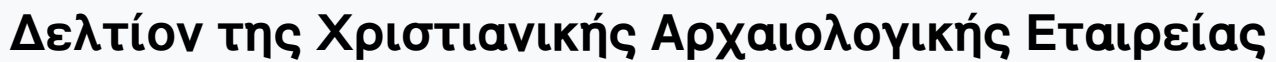

Tó 30 (2009)

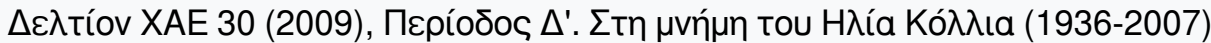

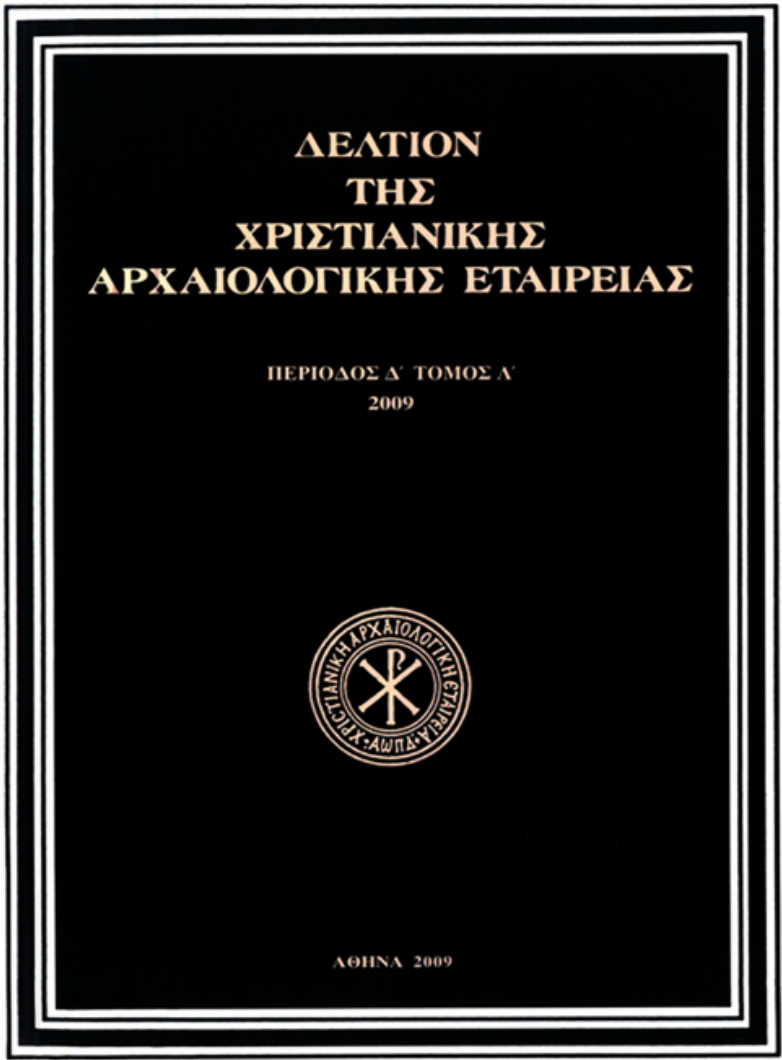

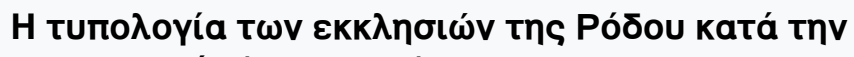
เппотократі́a (1309-1522)

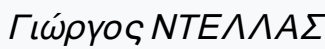

doi: $\underline{10.12681 / \text { dchae. } 638}$

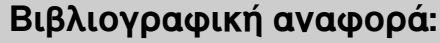

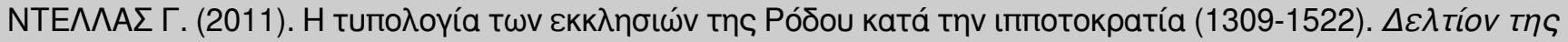

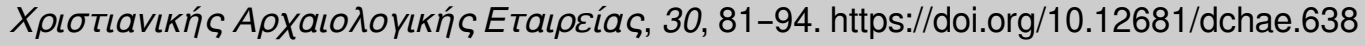




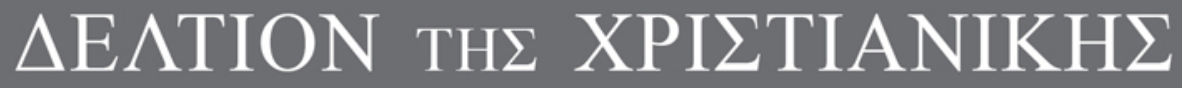 APXAIO $\Lambda$ OГIKH $\Sigma$ ETAIPEIA $\Sigma$}

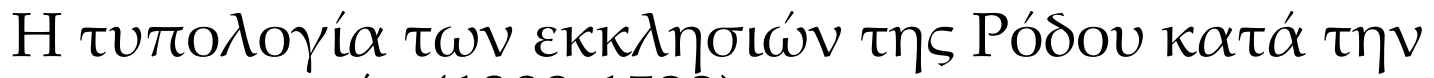
เттотокอ $\alpha \tau i ́ \alpha$ (1309-1522)

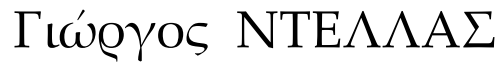

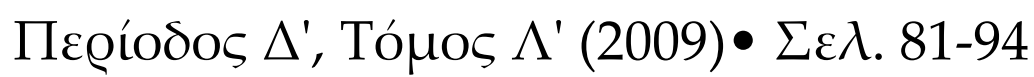
A@HNA 2009 


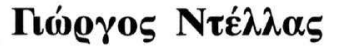

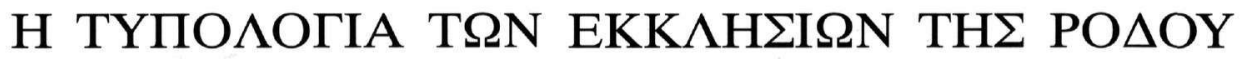 KATA THN IППОТОKPATIA (1309-1522)}

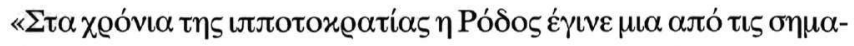

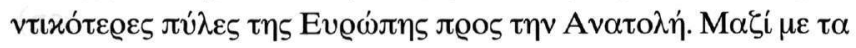

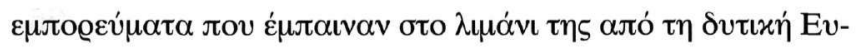

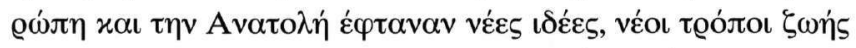

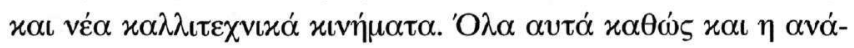

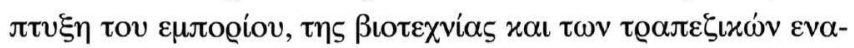

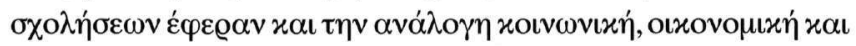

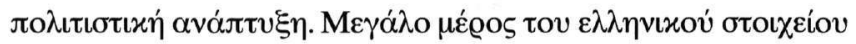

To

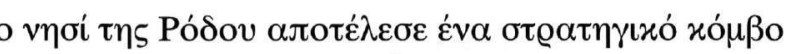

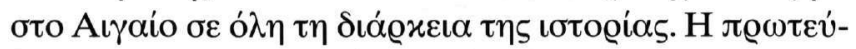

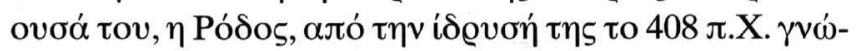

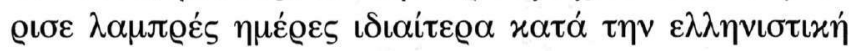

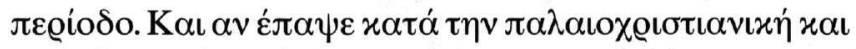

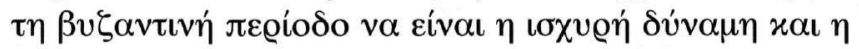

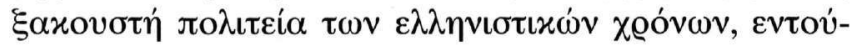

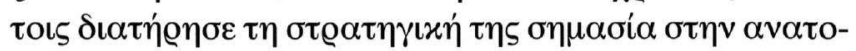

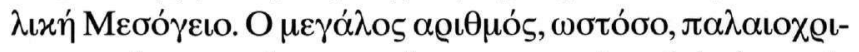

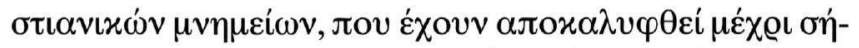

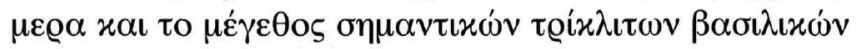

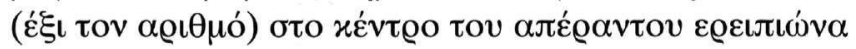

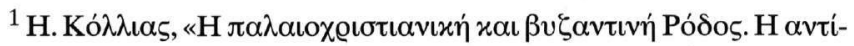

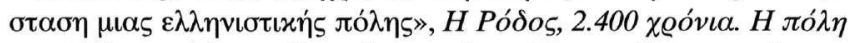

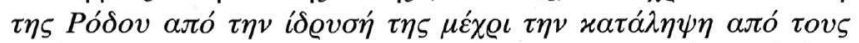

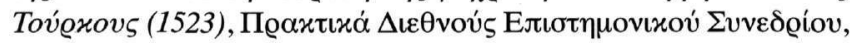

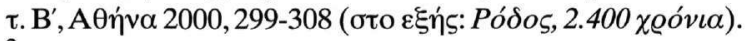

${ }^{2}$ E. Papavassiliou - Th. Archontopoulos, «Nouveaux éléments historiques et archéologiques de Rhodes à travers des fouilles dans la ville médiévale», CorsiRav XXXVIII (1991), 307-350. K. Mavov́oov - E.

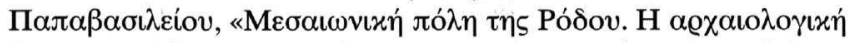

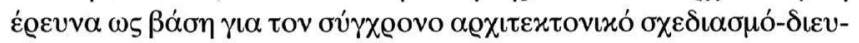

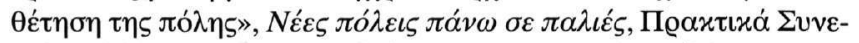

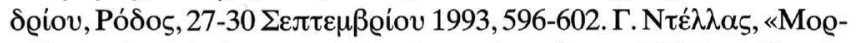

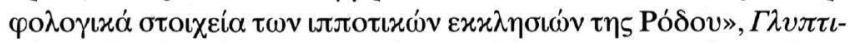

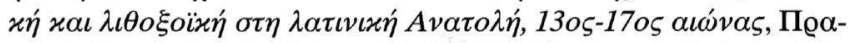

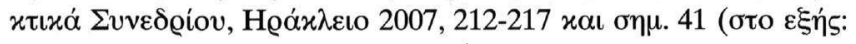

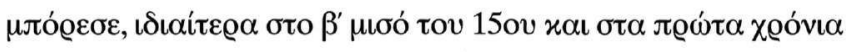

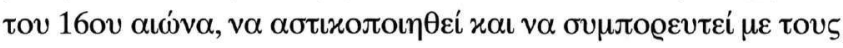

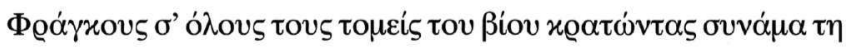


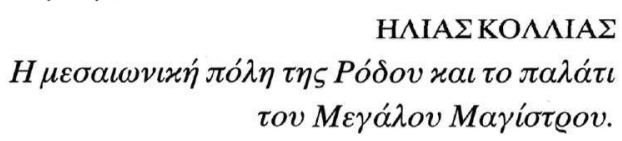

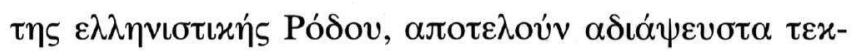

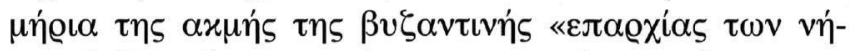

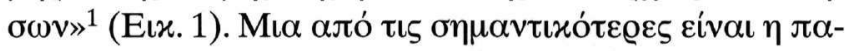

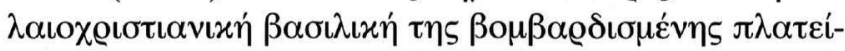

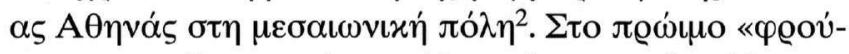

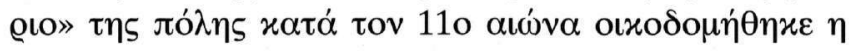

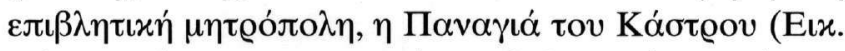

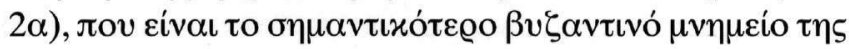

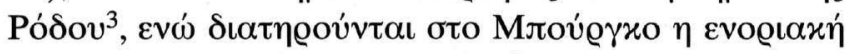

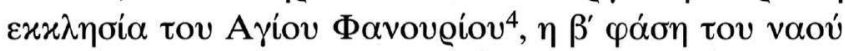

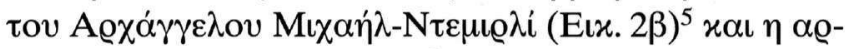

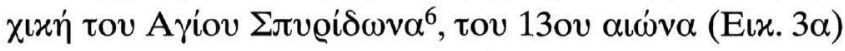

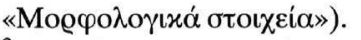

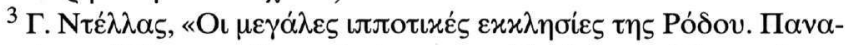

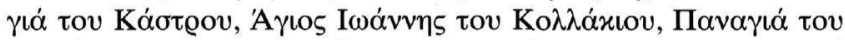

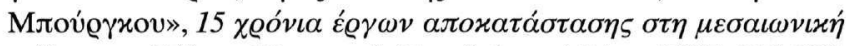

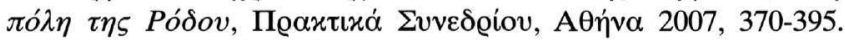

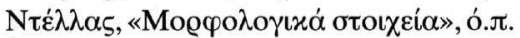

${ }^{4}$ A. Gabriel, La cité de Rhodes, Architecture civile et réligieuse, $\tau$. II, П $\alpha-$

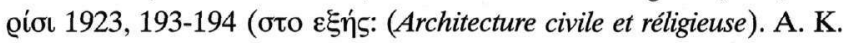

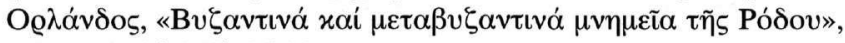
ABME $\Sigma \mathrm{T}^{\prime}$ (1948), 85-98.

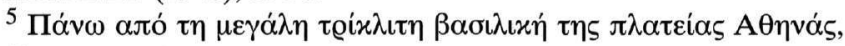

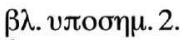

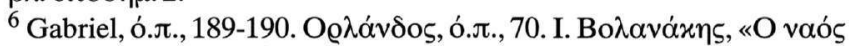

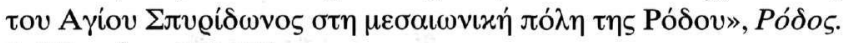

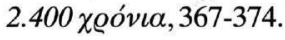




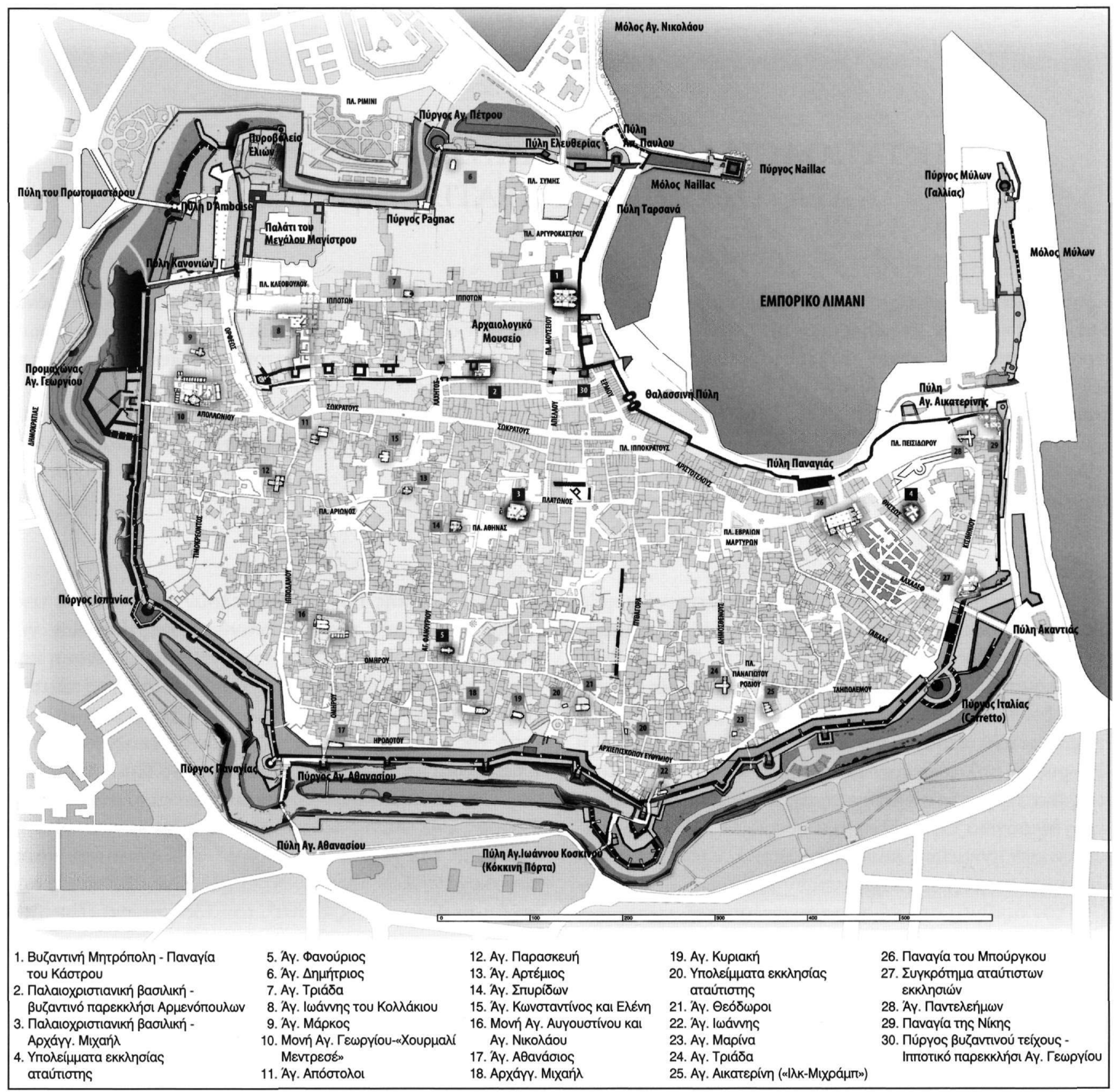

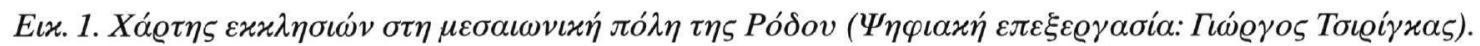

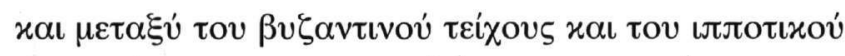

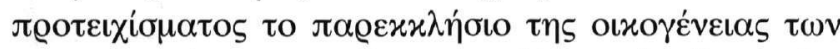

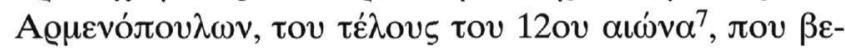

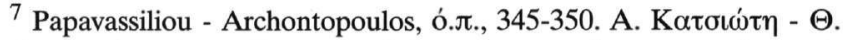

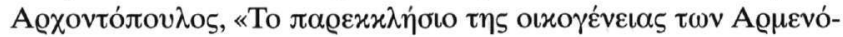

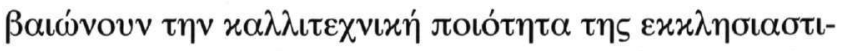

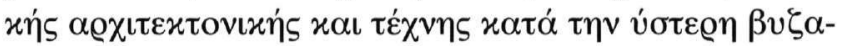

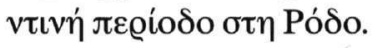

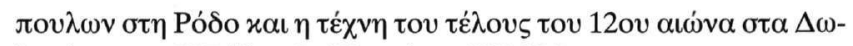

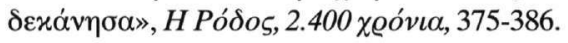




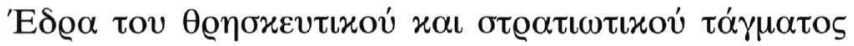

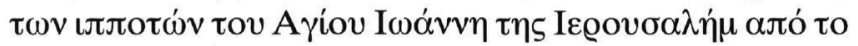

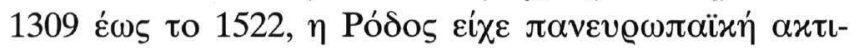

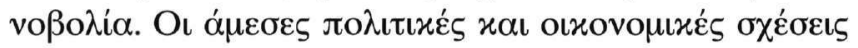

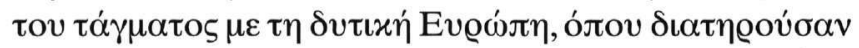

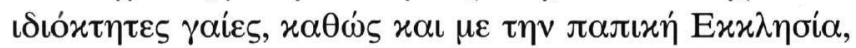

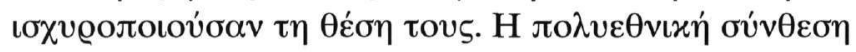

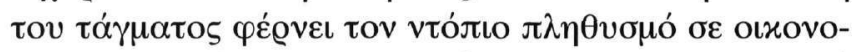

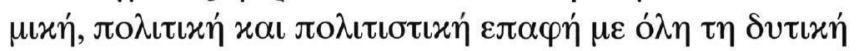

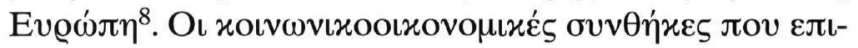

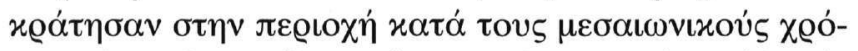

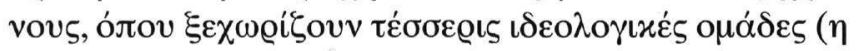

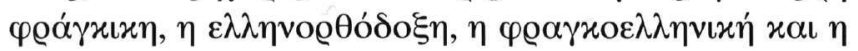

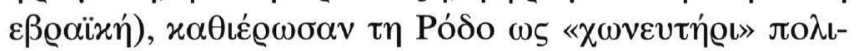

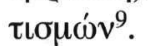

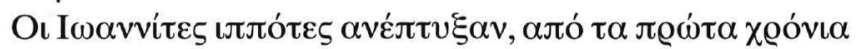

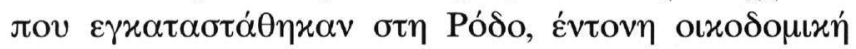

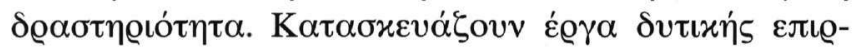

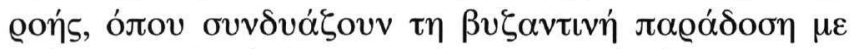

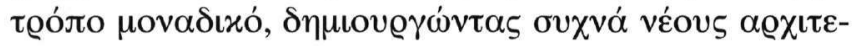

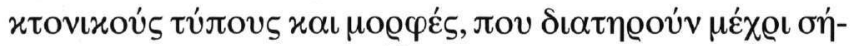

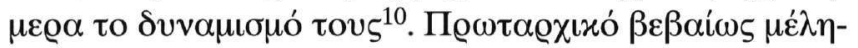

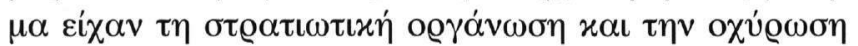

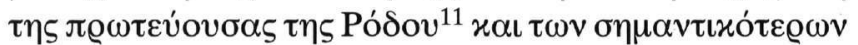

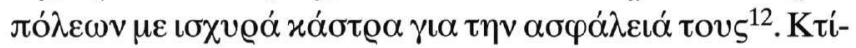

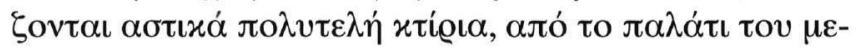

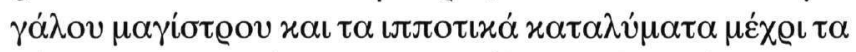

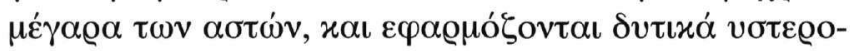

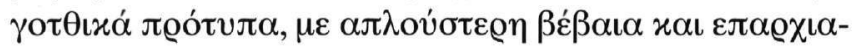

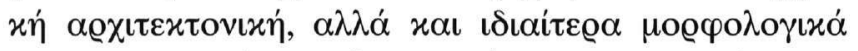

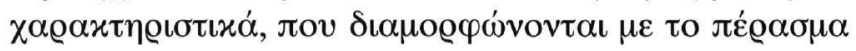

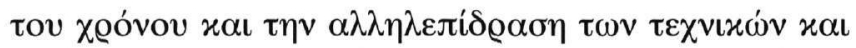

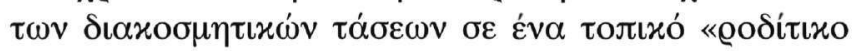

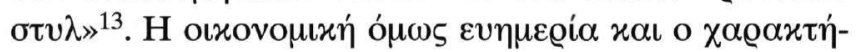

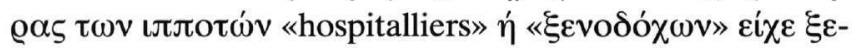

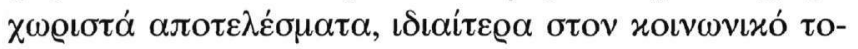

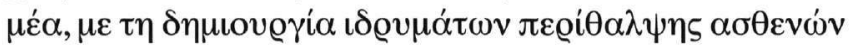

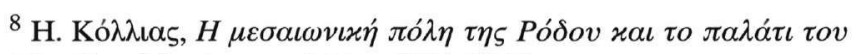

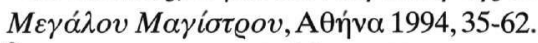

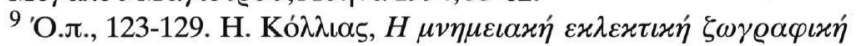

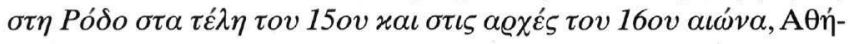
$v \alpha 2000$.

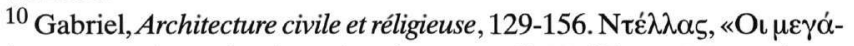

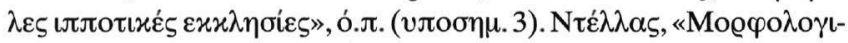

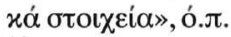

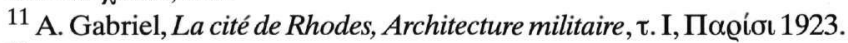

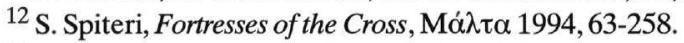

${ }^{13}$ Gabriel, Architecture civile et réligieuse, 1-128.
}
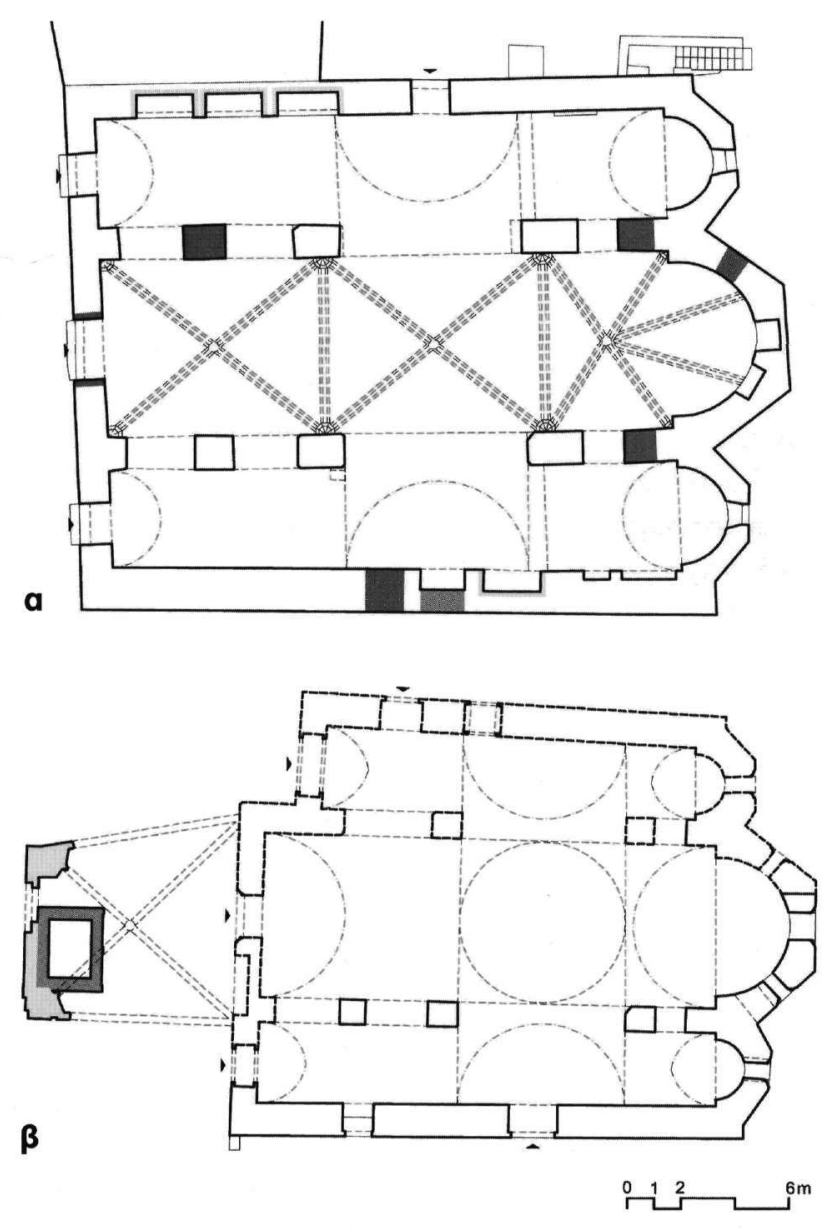

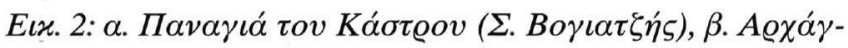

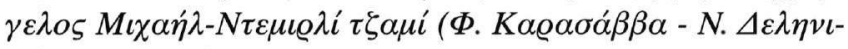

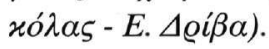

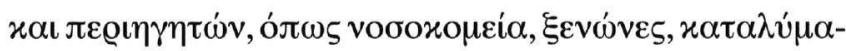
$\tau \alpha, \mu$ о $\alpha \sigma \tau \eta \dot{\rho} \alpha$.

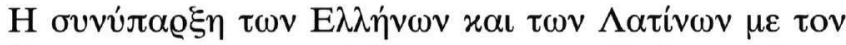

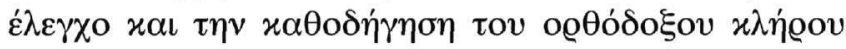

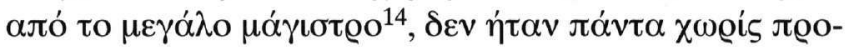

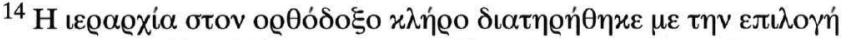

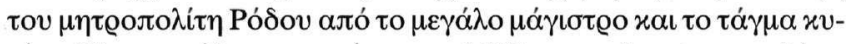

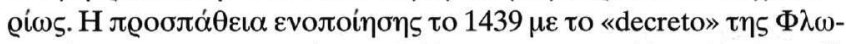

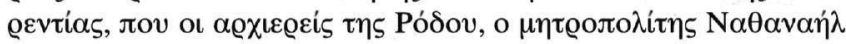

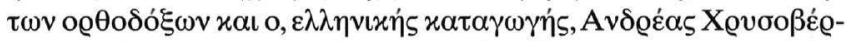

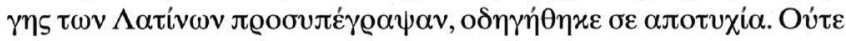

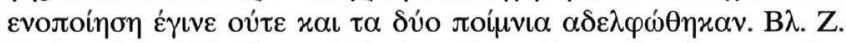

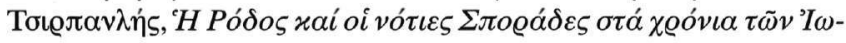

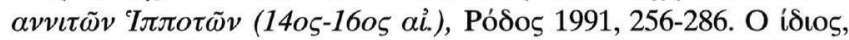

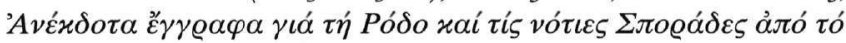

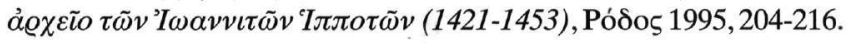




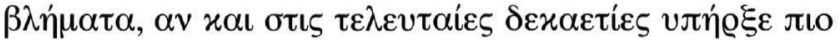

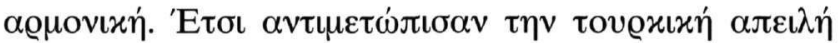

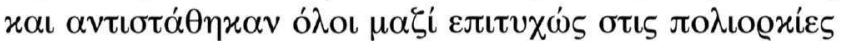

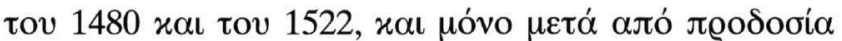

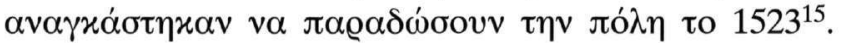

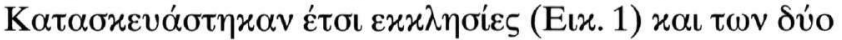

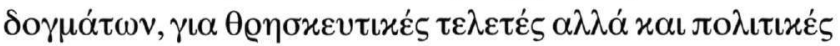

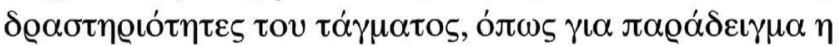

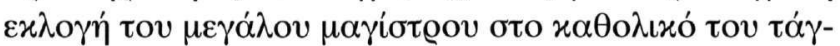

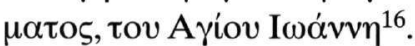

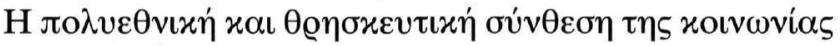

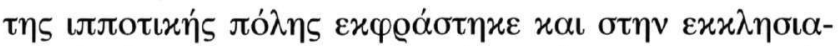

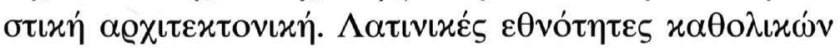

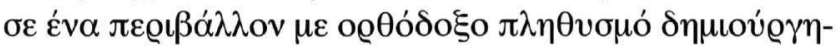

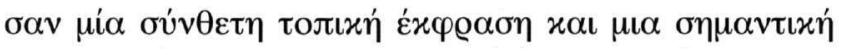

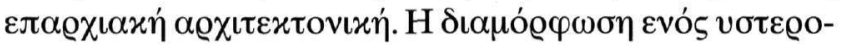

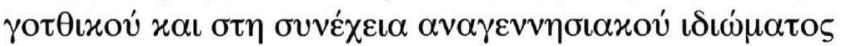

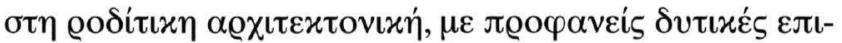

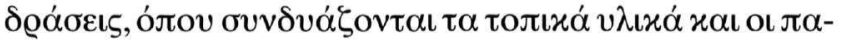

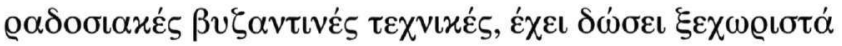

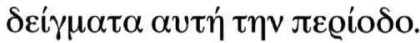

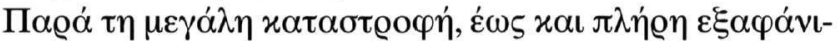

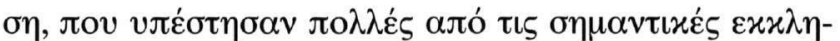

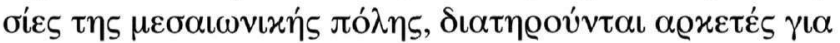

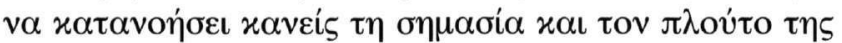

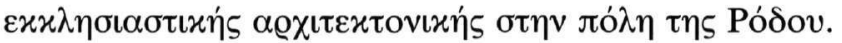

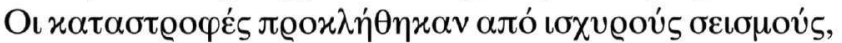

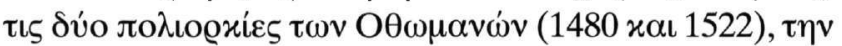

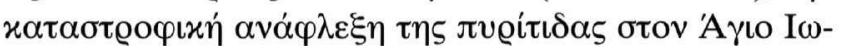

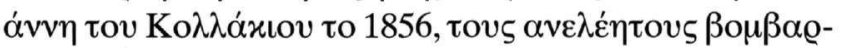

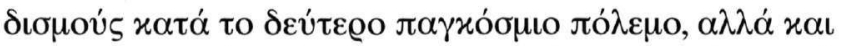

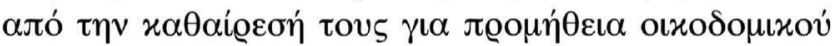
$v \lambda เ x o v^{17}$

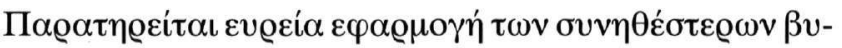

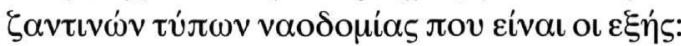

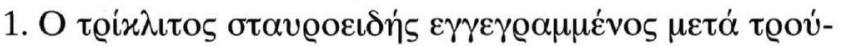

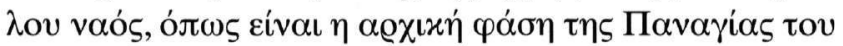

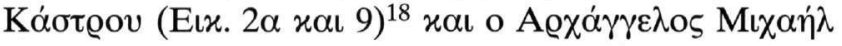

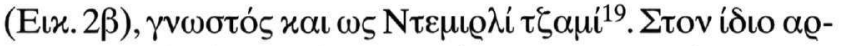

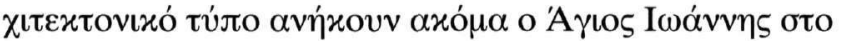

\footnotetext{
15 J. Bosio, Historia della sacra religione et illustrissima militia di San Giovanni Gerosolimitano e dell'ordine militare di San Sepolcro, $\tau$. II, P $\omega \mu \eta$

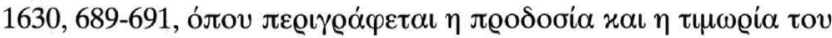

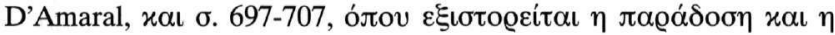

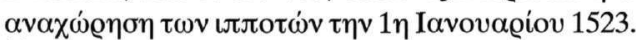

16 'O.л., 362 .

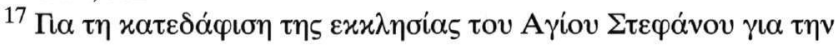
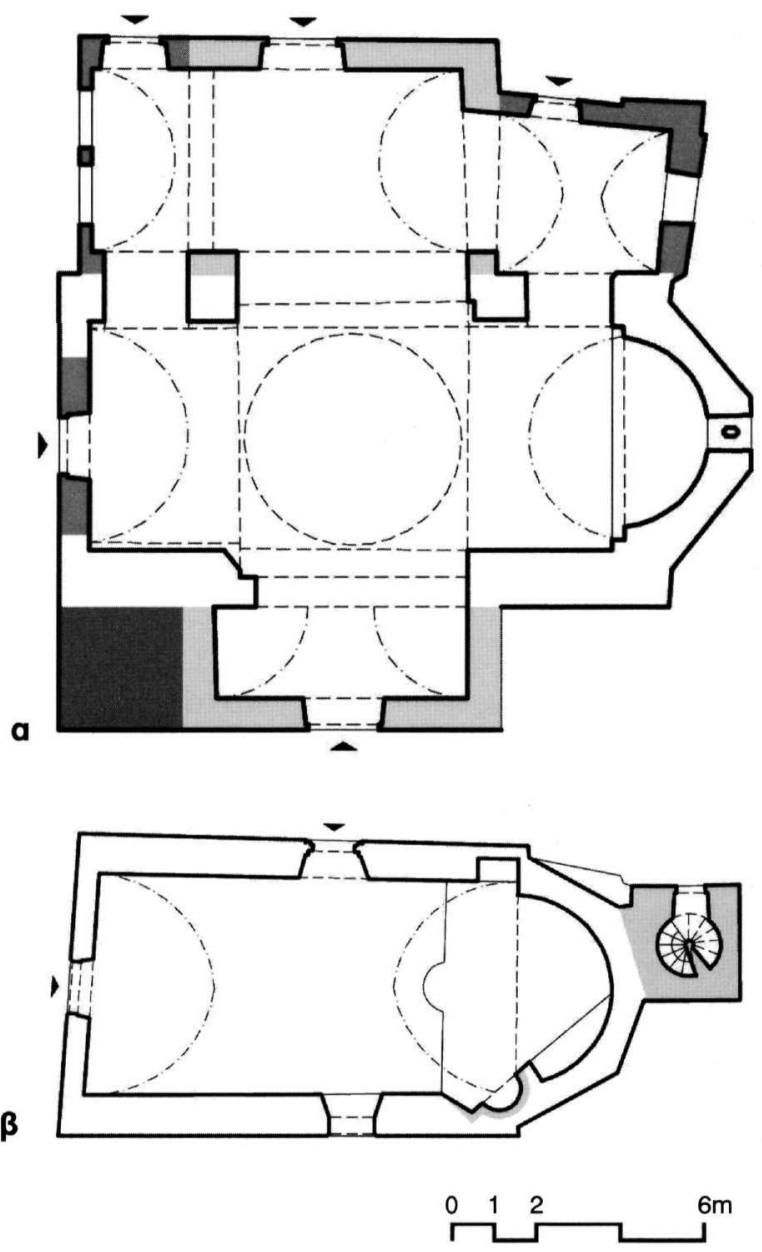

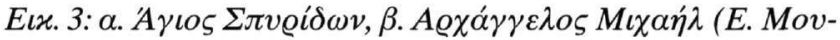
$\sigma \tau \alpha \dot{x} x)$.

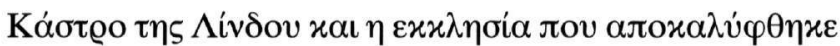

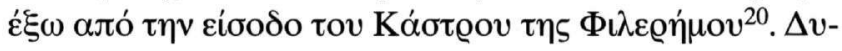

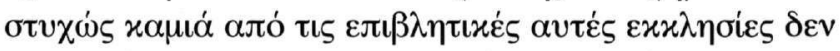

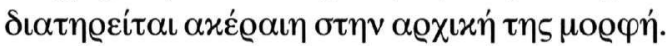

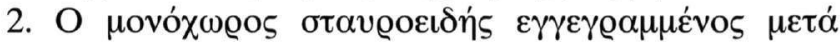

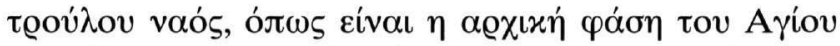

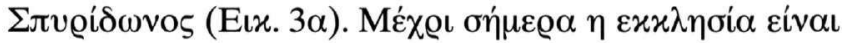

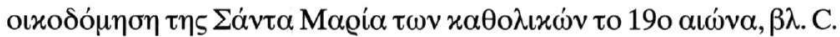
Torr, Rhodes in Modern Times, Cambridge 1887, 42-43.

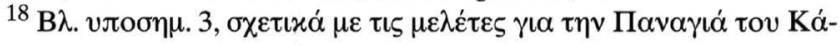

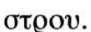

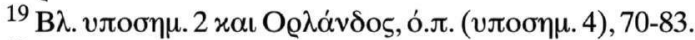

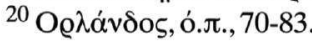




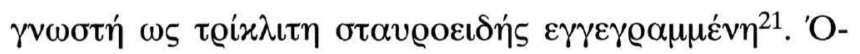

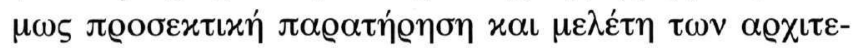

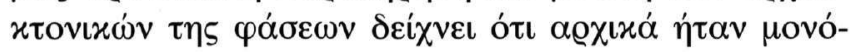

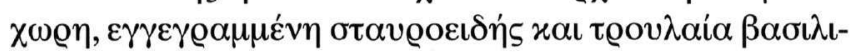

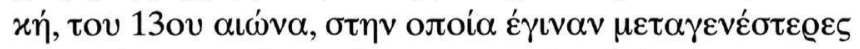

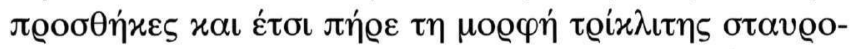

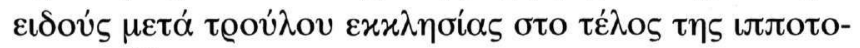

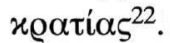

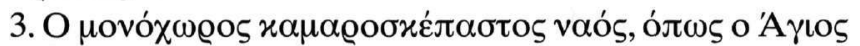

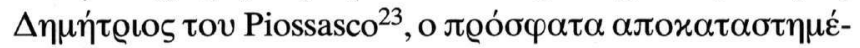

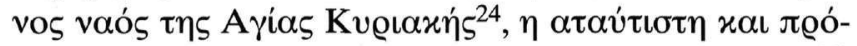

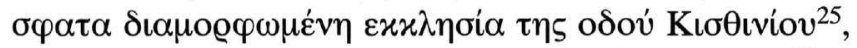

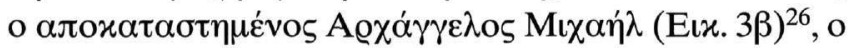

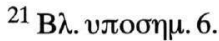

${ }^{22}$ Avód

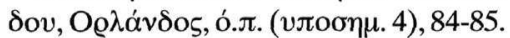

${ }^{23}$ M. Cante, «La cappella di S. Demetrio dei Piossasco a Rodi», H Pó-
}

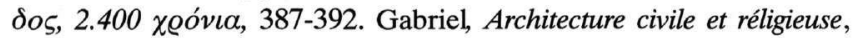

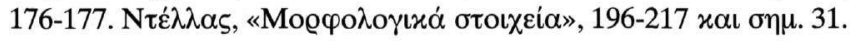

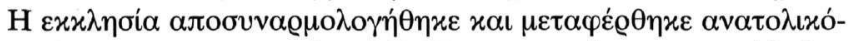

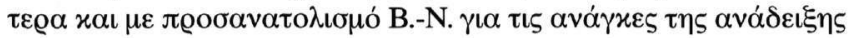

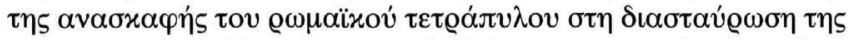

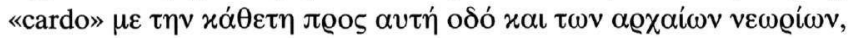

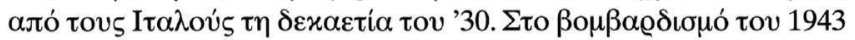

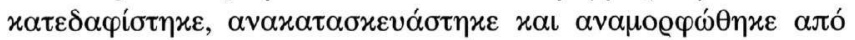

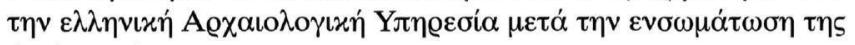
$\triangle \omega \delta \varepsilon x \alpha v \eta \dot{\sigma o v . ~}$

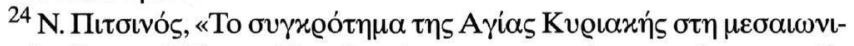

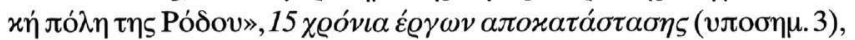

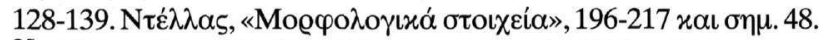

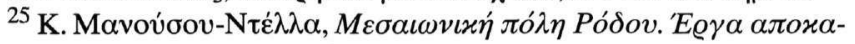

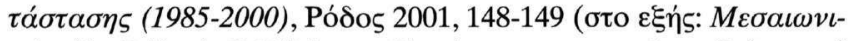

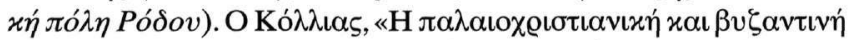

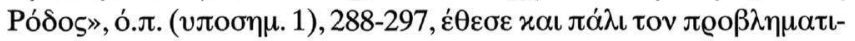

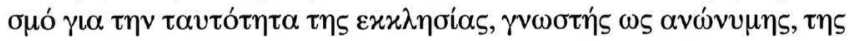

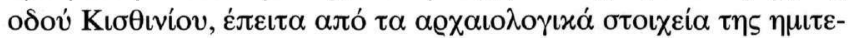

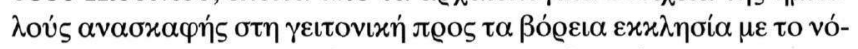

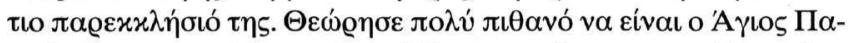

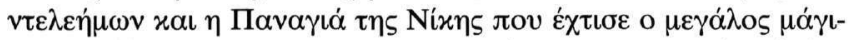

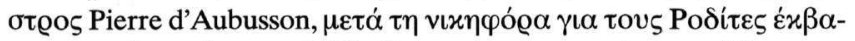

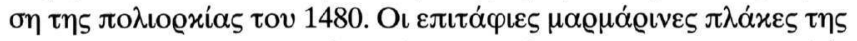

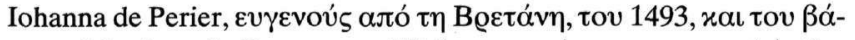

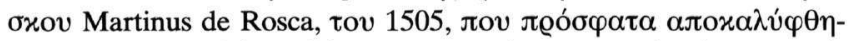

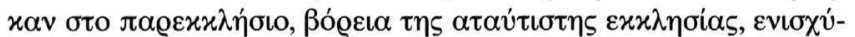

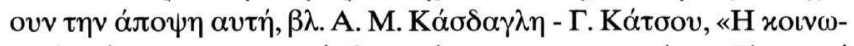

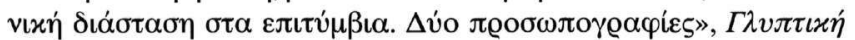

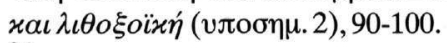

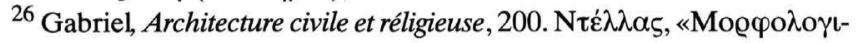

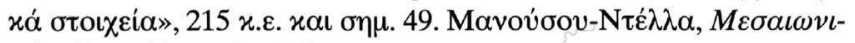

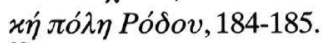

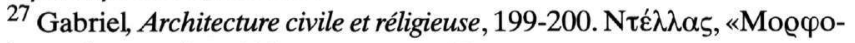

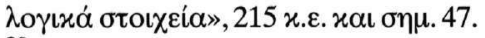

${ }^{28}$ Gabriel, Architecture civile et réligieuse, 202.

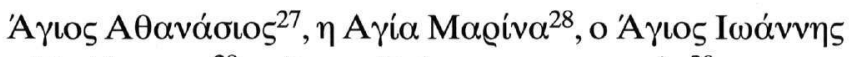

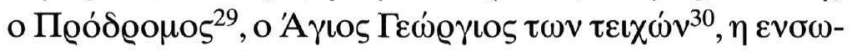

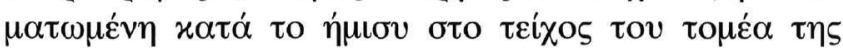

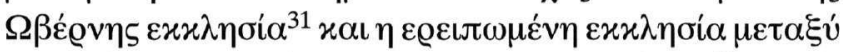

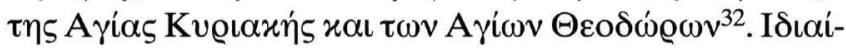

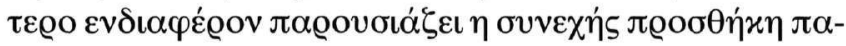

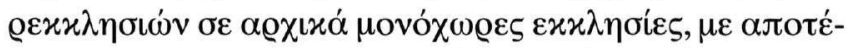

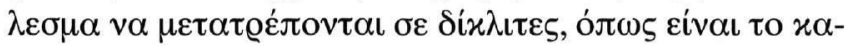

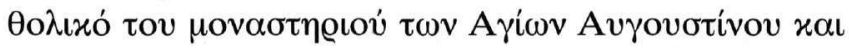

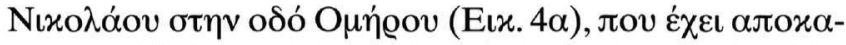

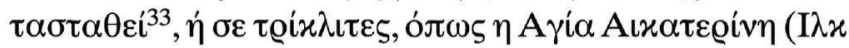

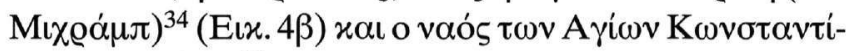

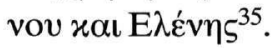

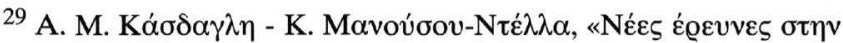

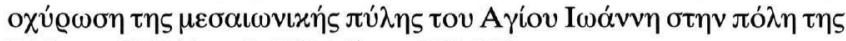

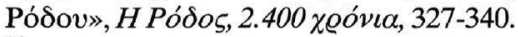

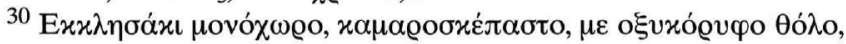

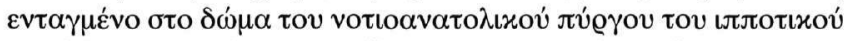

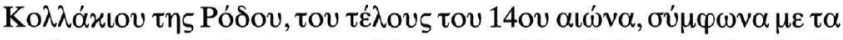

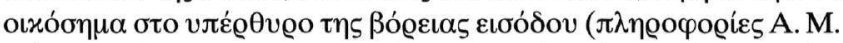

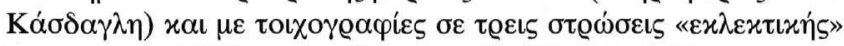

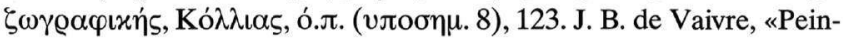
tures murales disparues ou en péril d'anciennes chapelles de l'ordre des hospitaliers», CRAI 2006, 1037-1065.

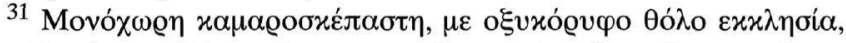

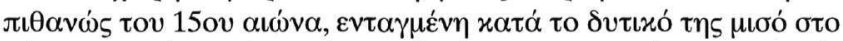

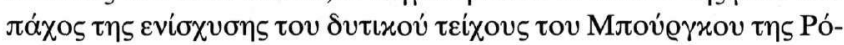

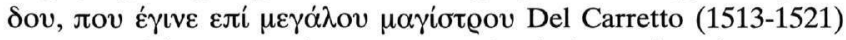

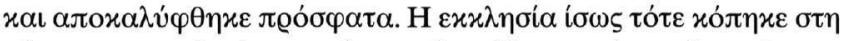

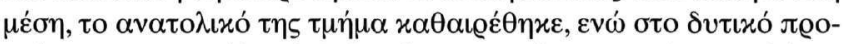

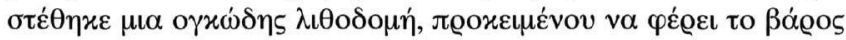

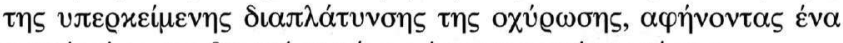

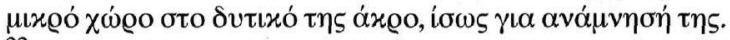

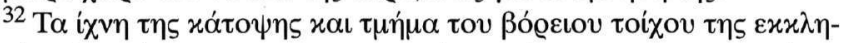

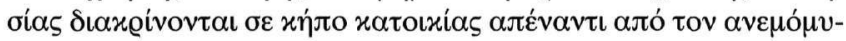

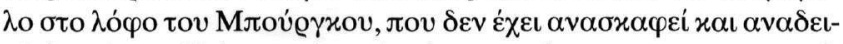

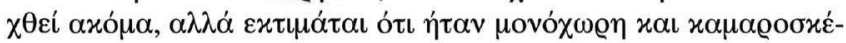
$\pi \alpha \sigma \tau \eta$.

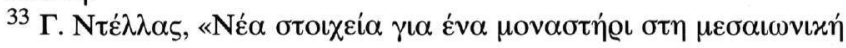

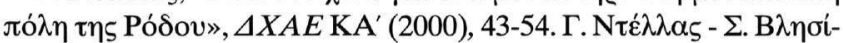

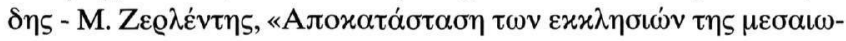

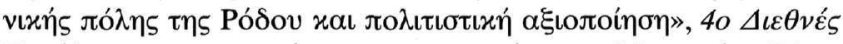

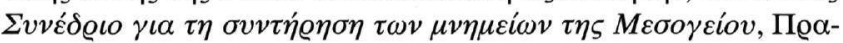

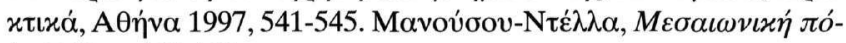
$\lambda \eta$ Pódov, 180-181.

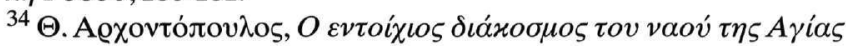

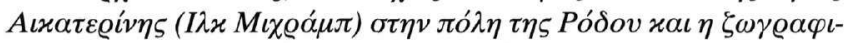

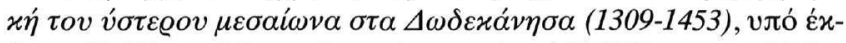

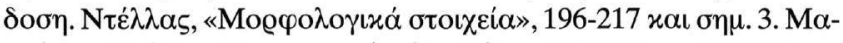

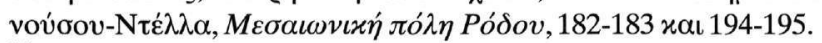

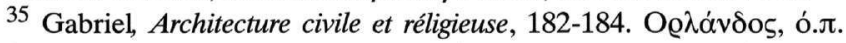

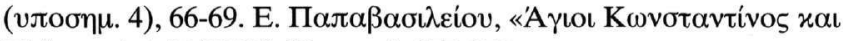

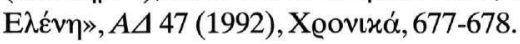



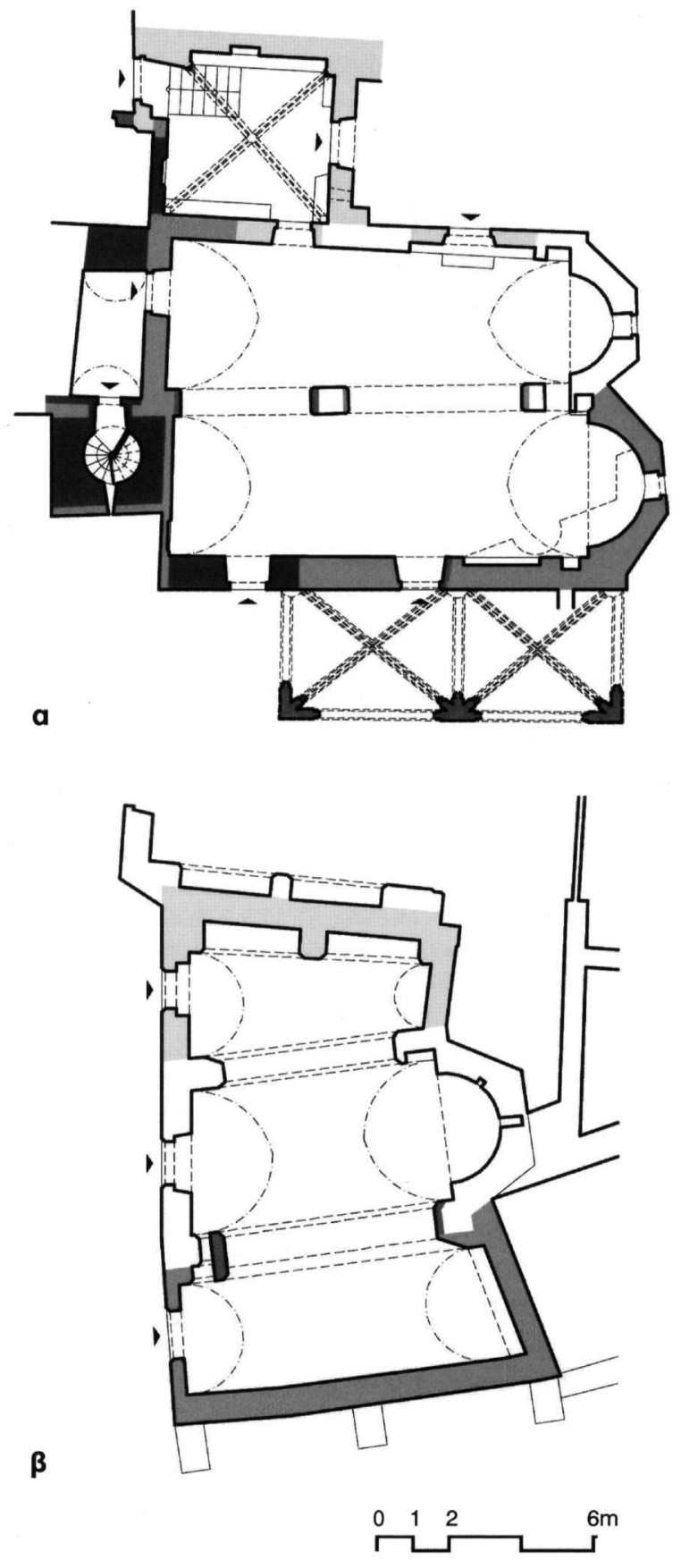

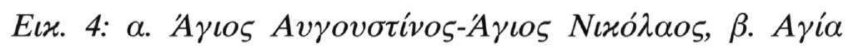

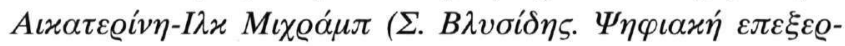

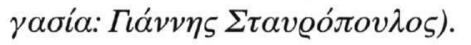

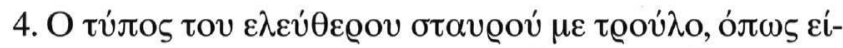

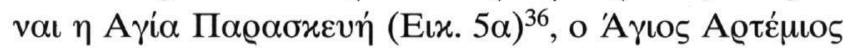

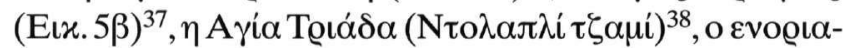

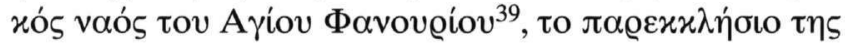

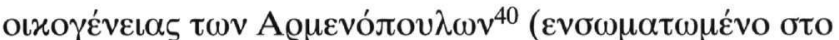

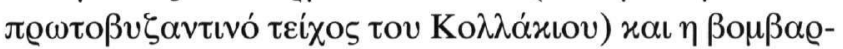

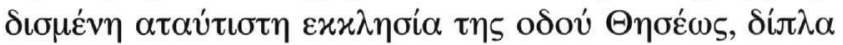

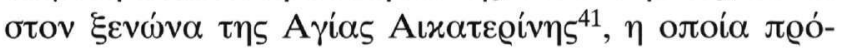

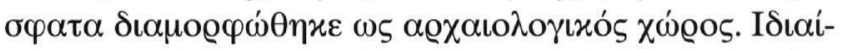

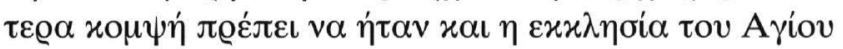

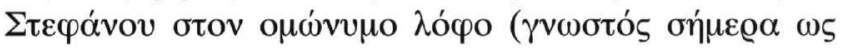

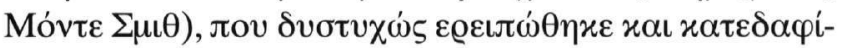

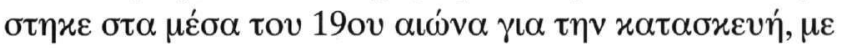

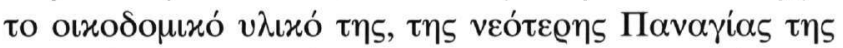

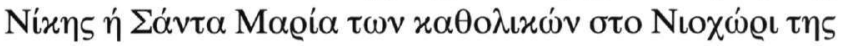

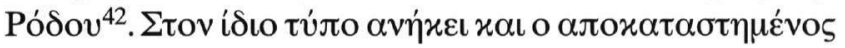
A

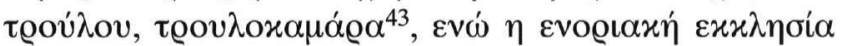

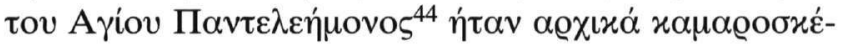

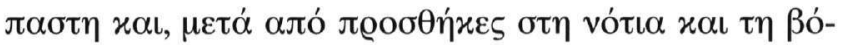

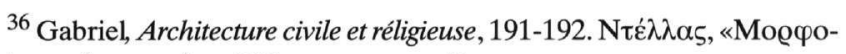

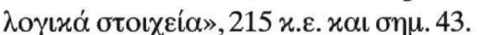

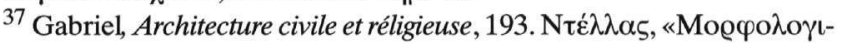

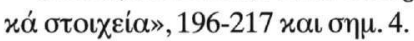

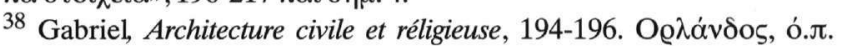

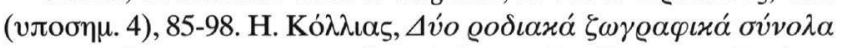

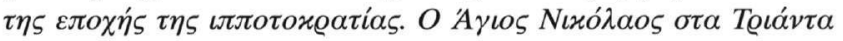

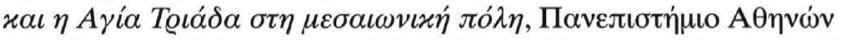

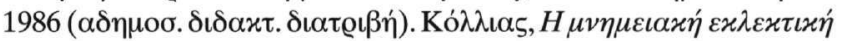

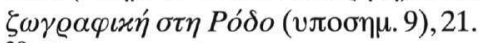

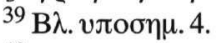

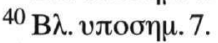

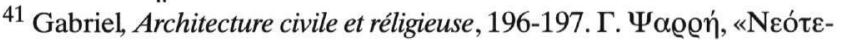

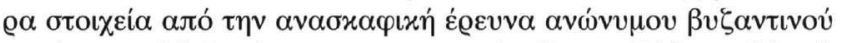

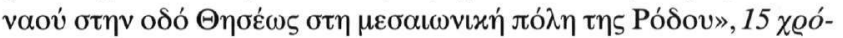

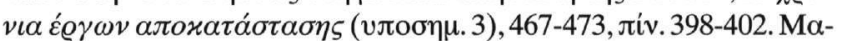

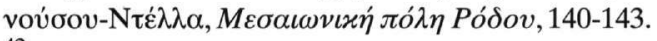

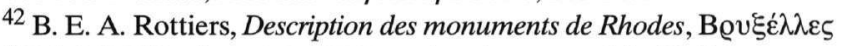

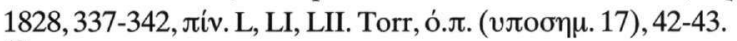

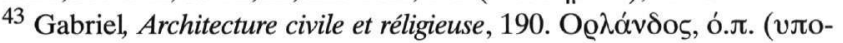

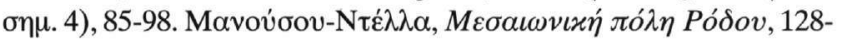
129.

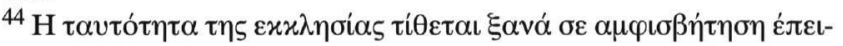

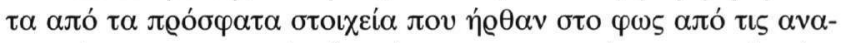

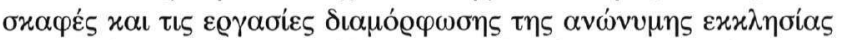

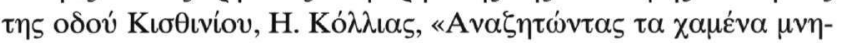

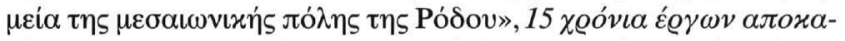

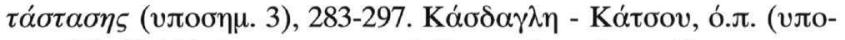

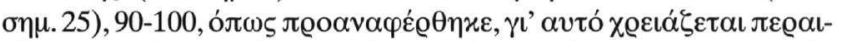

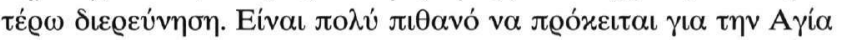

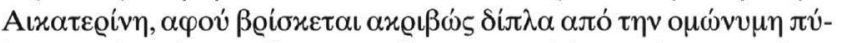

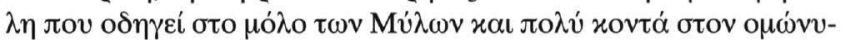

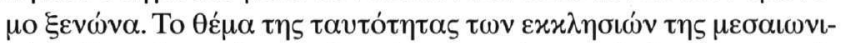

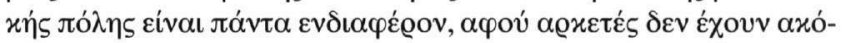

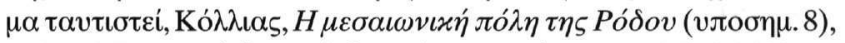

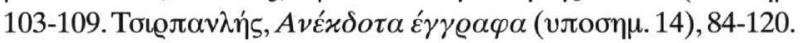



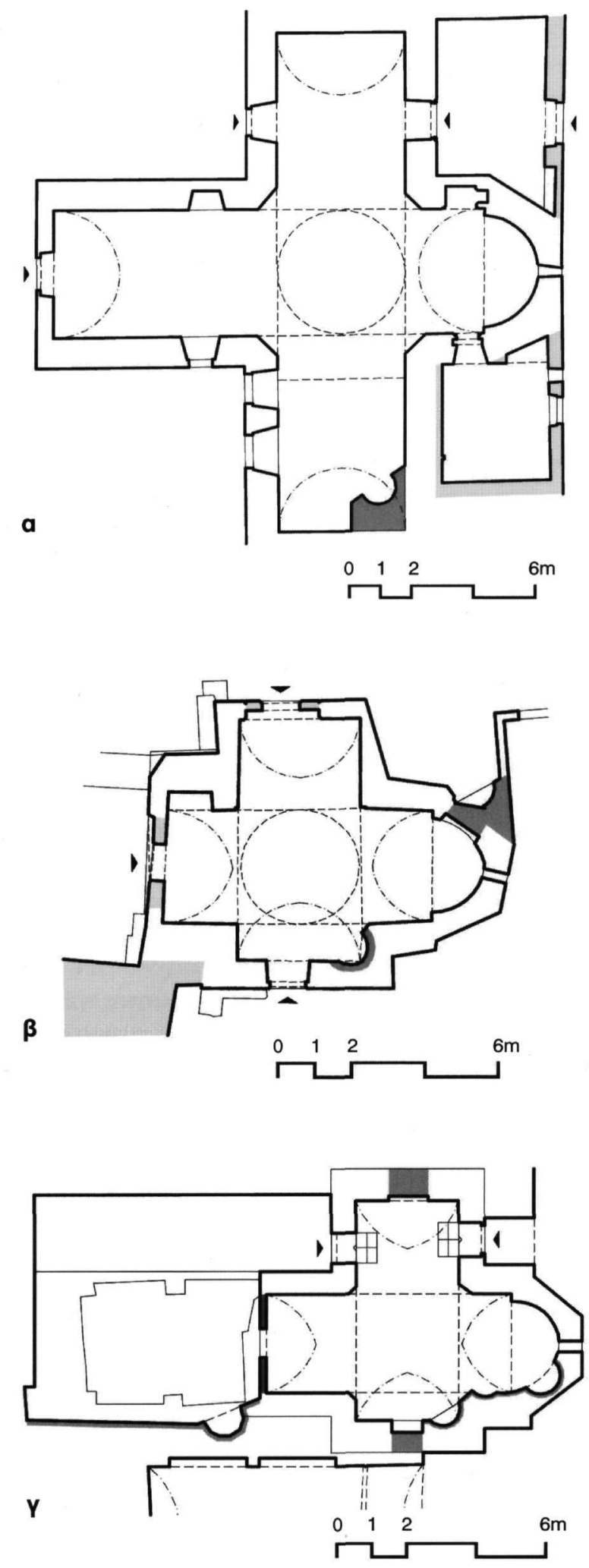

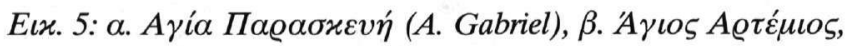

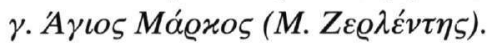

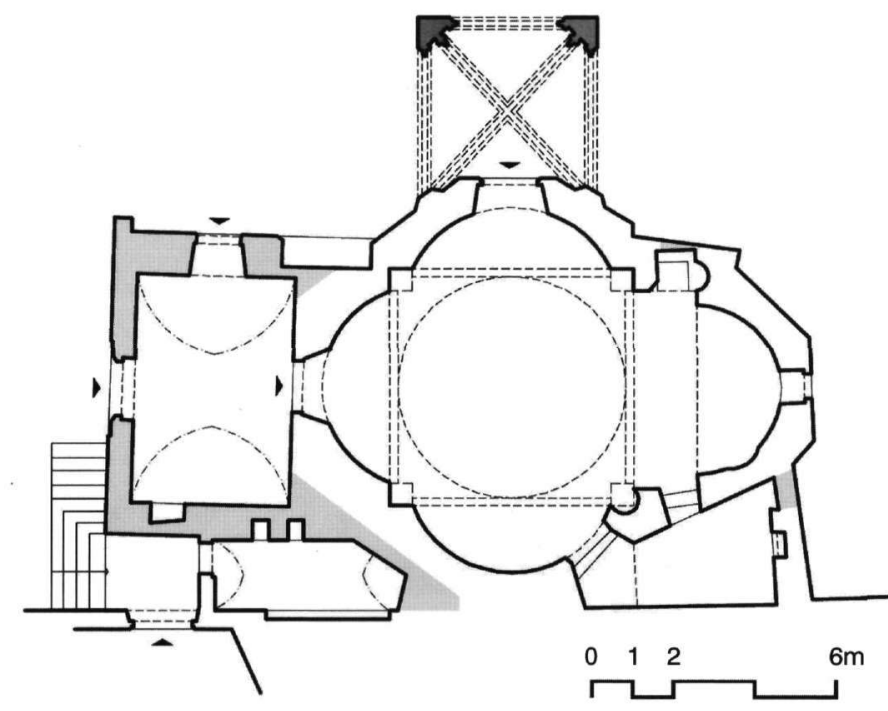

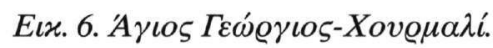

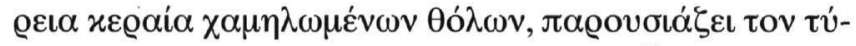

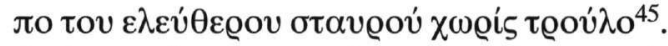

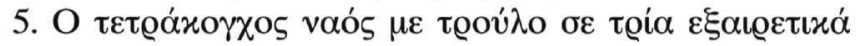

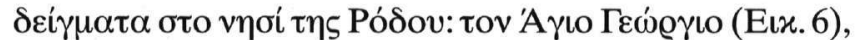

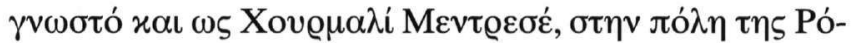

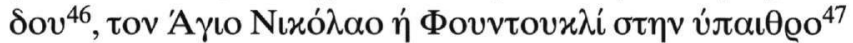

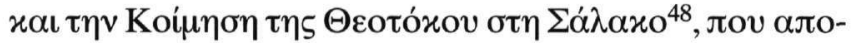

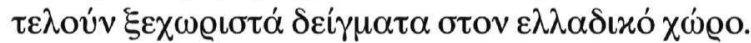

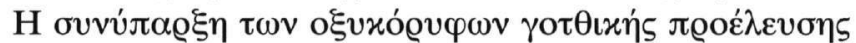

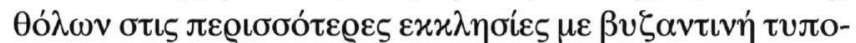

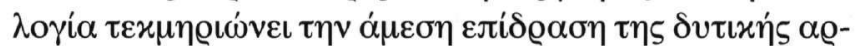

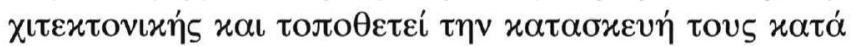

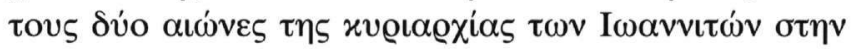

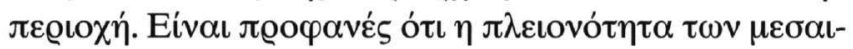

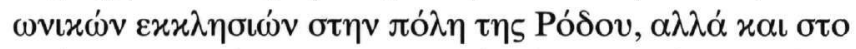

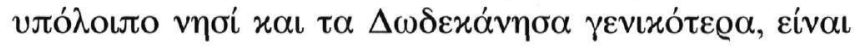

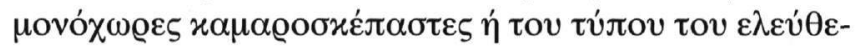

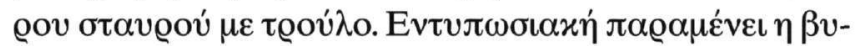

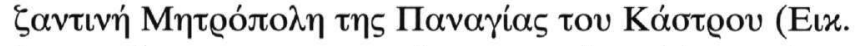

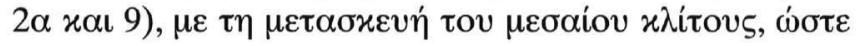

\footnotetext{
${ }^{45}$ Gabriel, Architecture civile et réligieuse, 197-198.

46 'O.л., 202-207. H. Balducci, Architettura turca in Rodi, Milano 1932,

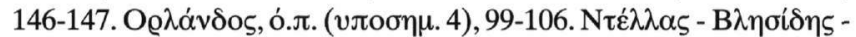

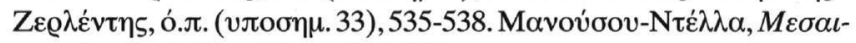

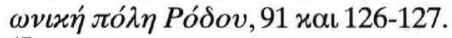

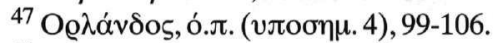

48 'О.л.
} 

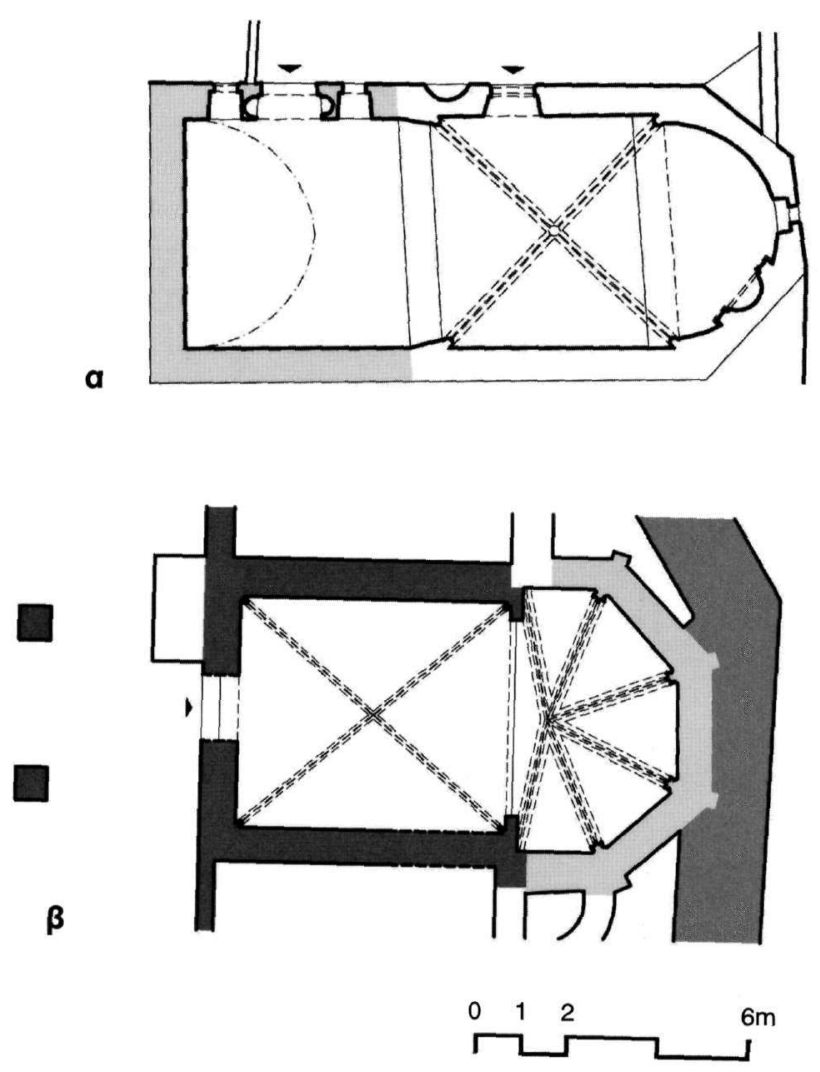

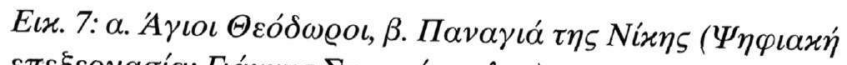

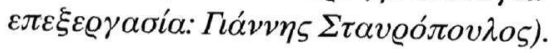

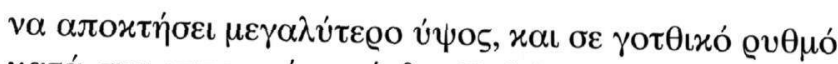

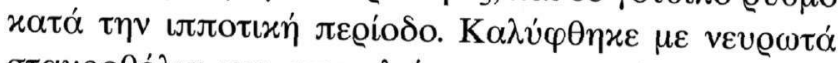

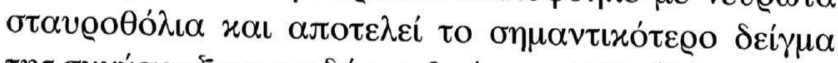

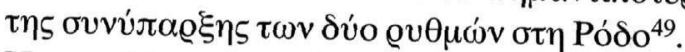

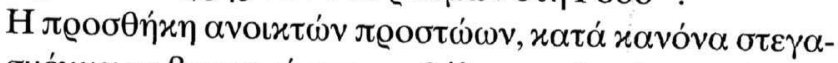

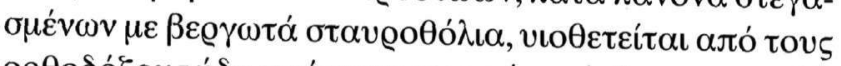

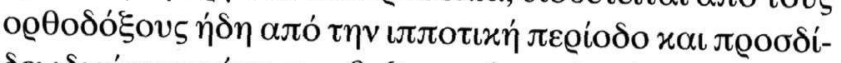

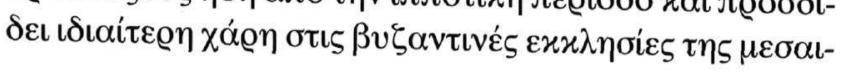

\footnotetext{
${ }^{49} \mathrm{~B} \lambda$. vлобпн. 3.

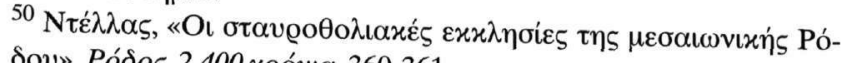

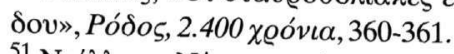

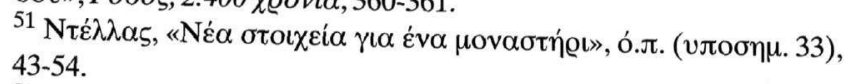

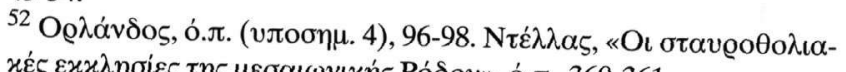

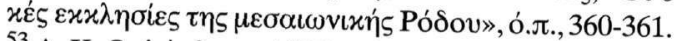

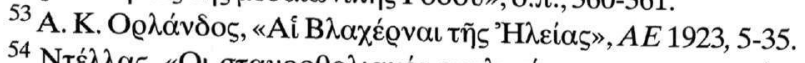

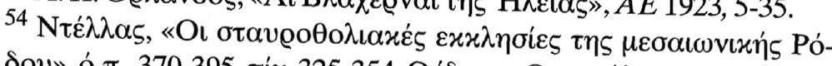

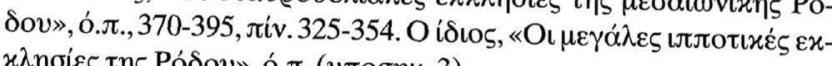

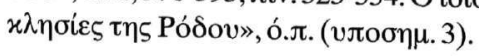

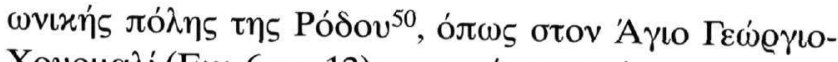

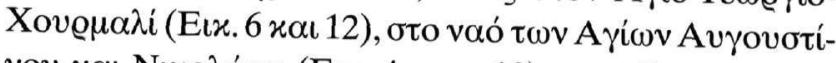

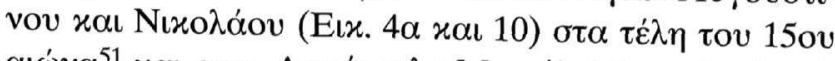

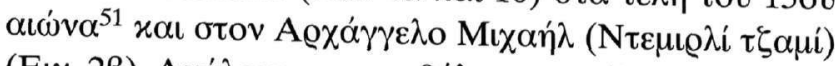

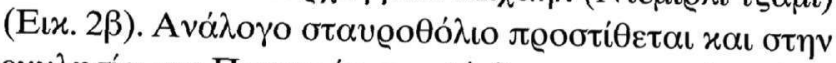

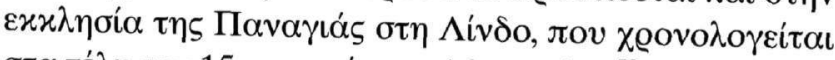

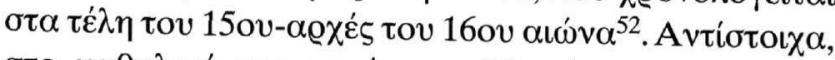

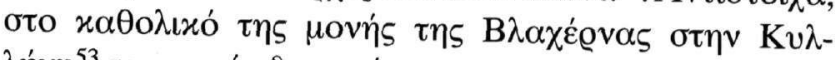

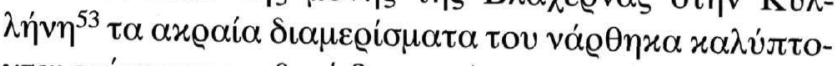

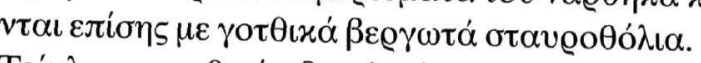

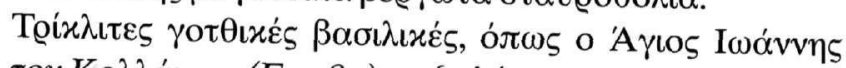

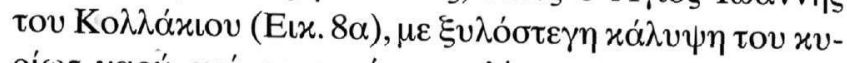

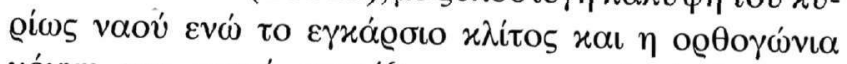

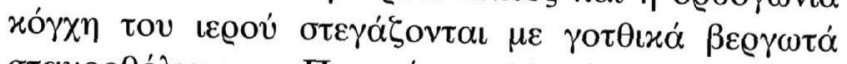

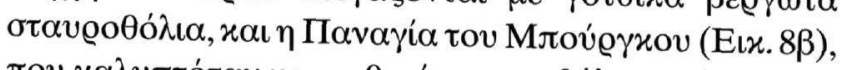

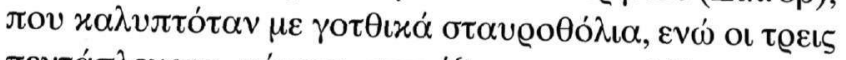

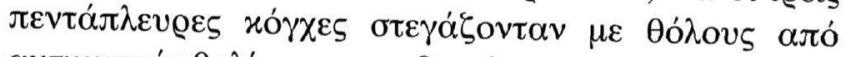

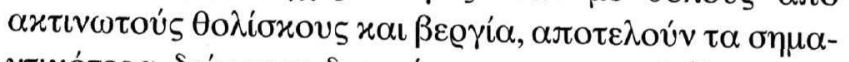

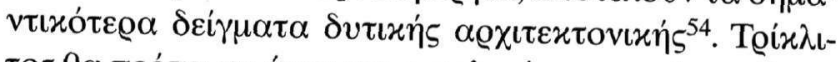

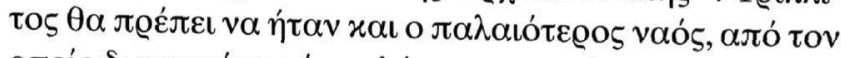

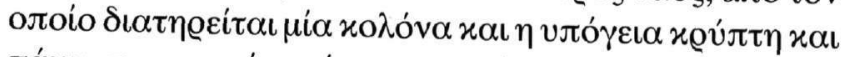

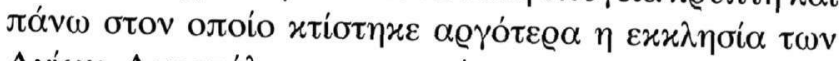

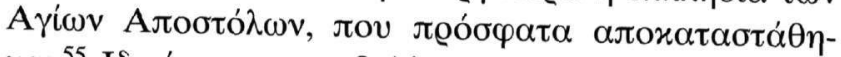

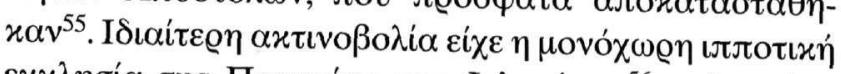

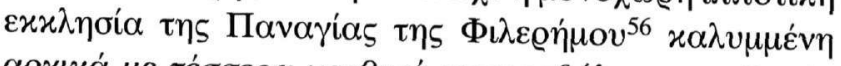

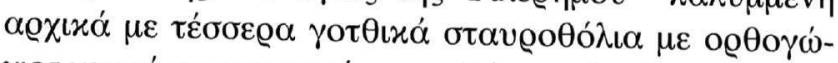

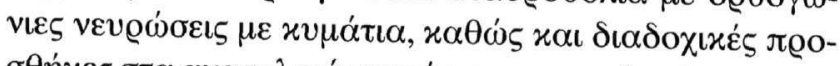

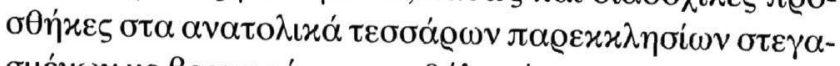

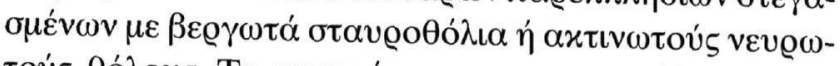

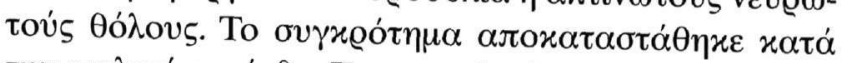

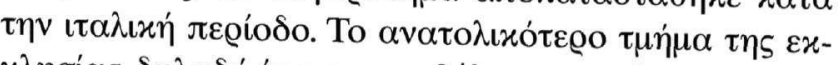

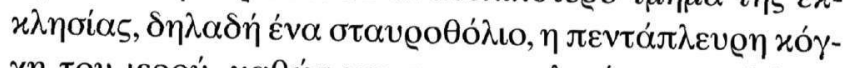

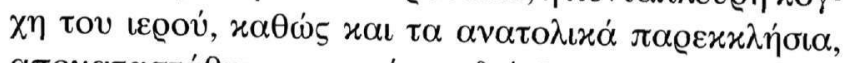

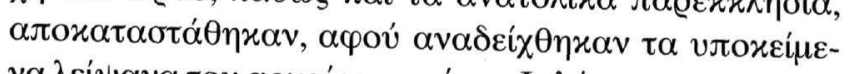

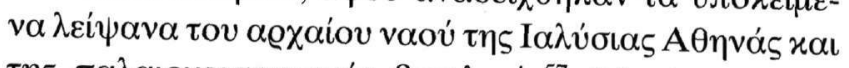

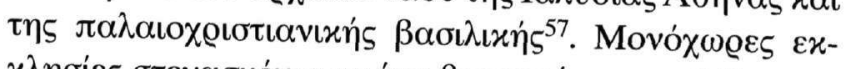

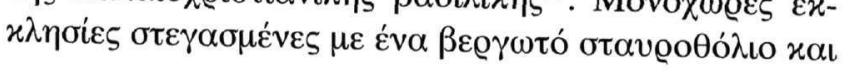

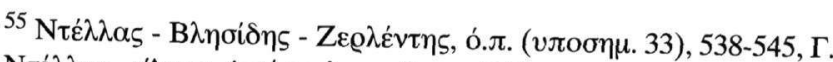

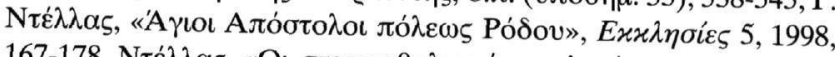

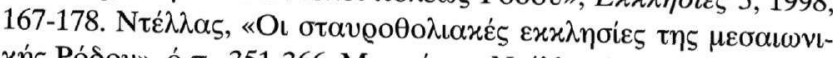

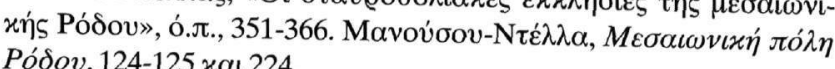
Pódov, 124-125 xal 224.

${ }^{56}$ H. Balducci, Il santuario di N. Signora di Tutte le Grazie sul Filerimo

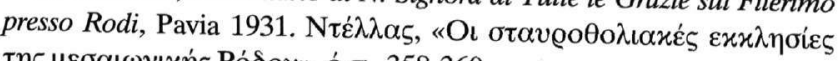

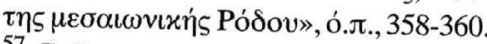

57 G. Rocco, «Il restauro del santuario di Nostra Signora di Tutte le Grazie», La presenza italiana nel Dodecaneso tra il 1912 e il 1948, A $\theta \dot{\eta}-$
va 1997, 261-265.
} 

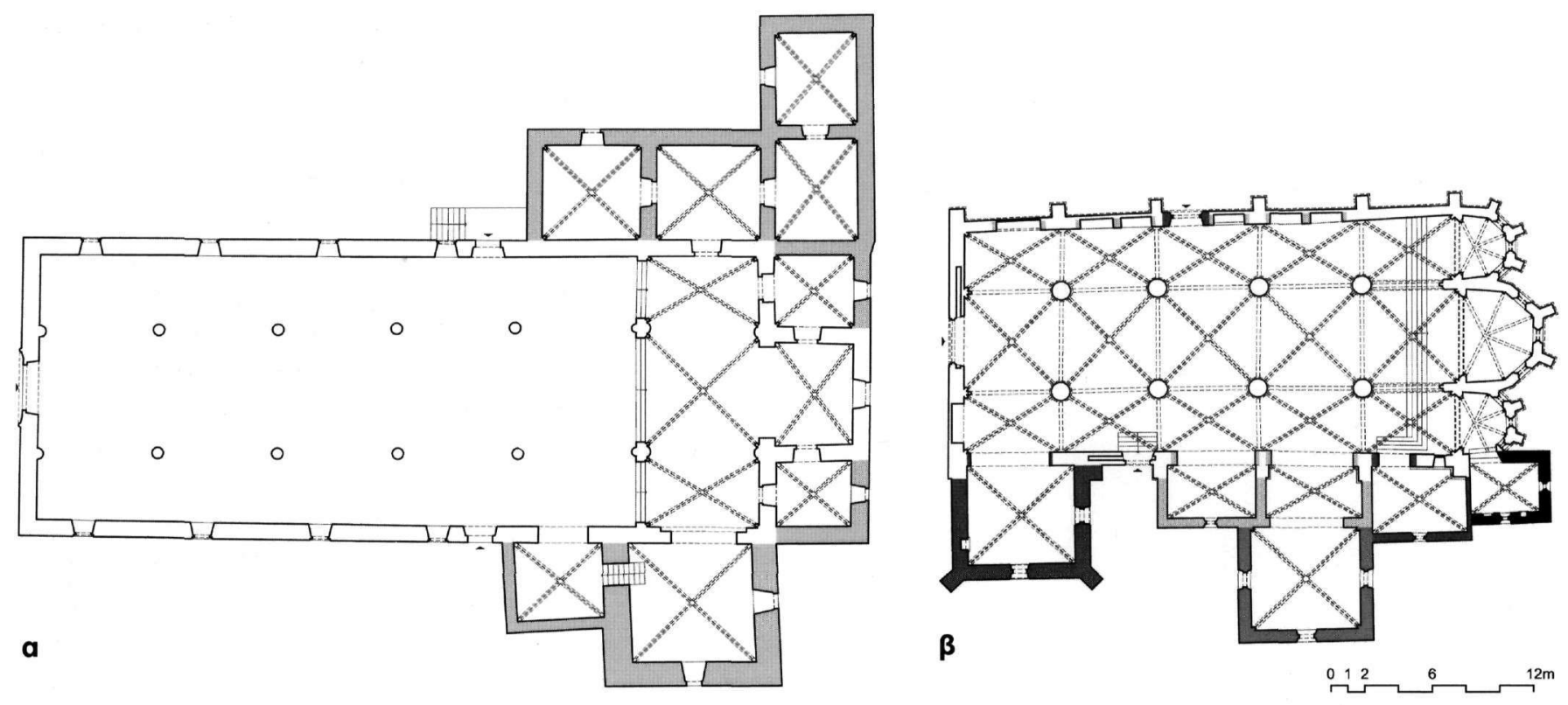

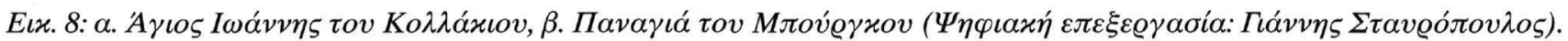

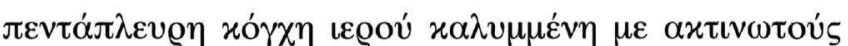

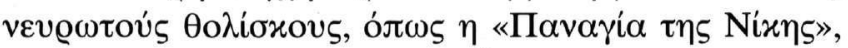

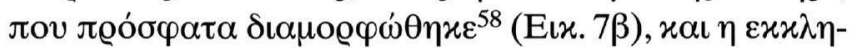

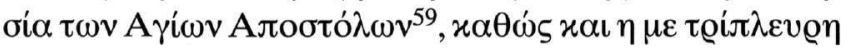

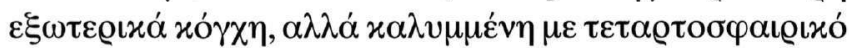

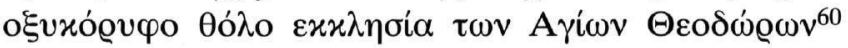

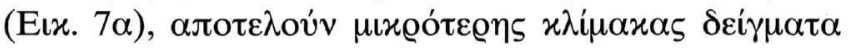

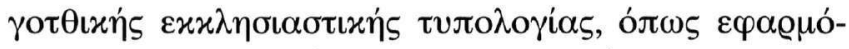

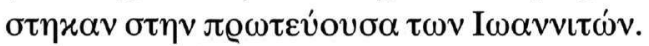

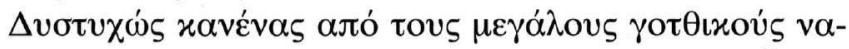

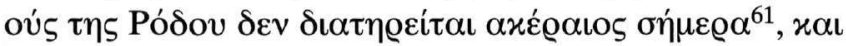

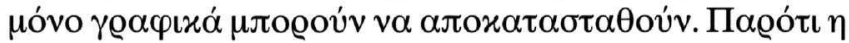

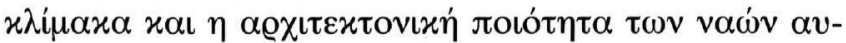

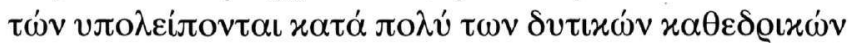

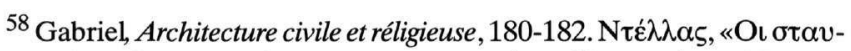

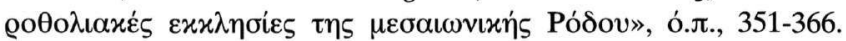

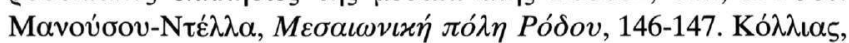

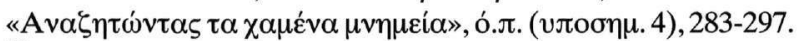

${ }^{59} \mathrm{~B} \lambda$. ขлобпu. 55.

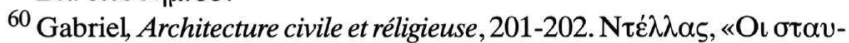

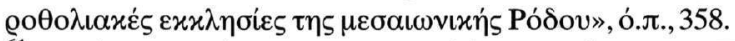

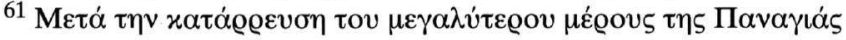

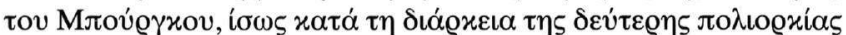

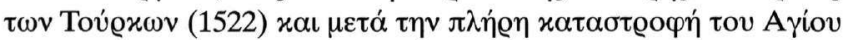

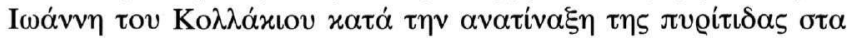

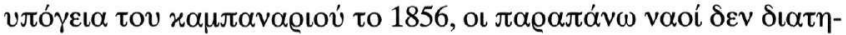

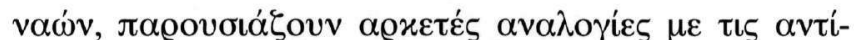

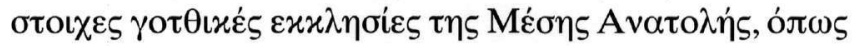

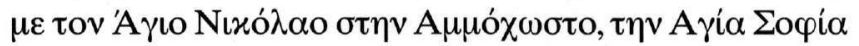

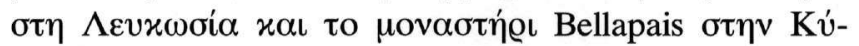

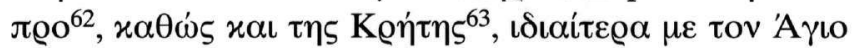

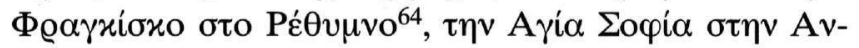

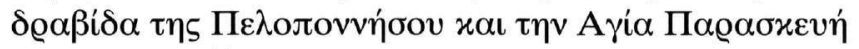
$\sigma \tau \eta \mathrm{X} \alpha \lambda x i \delta \alpha^{65}$.

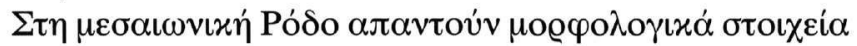

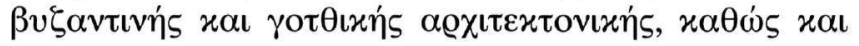

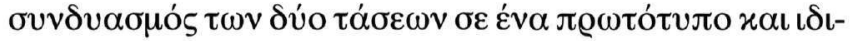

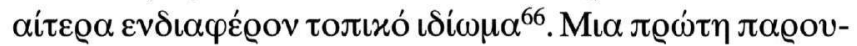

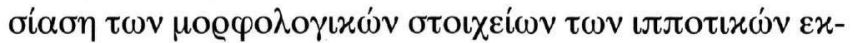

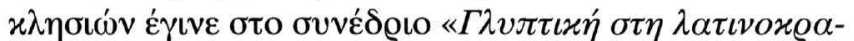

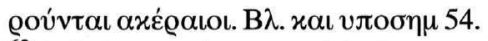

${ }^{62}$ C. Enlart, Gothic Art and the Renaissance in Cyprus, Па@íı 1899, 82 131, 174-200, 222-245.

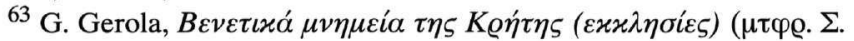

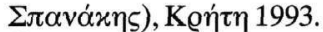

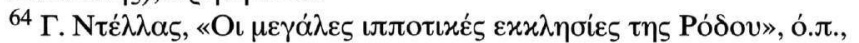

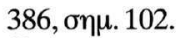

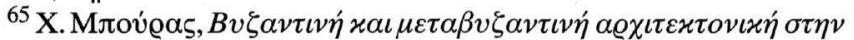
$E \lambda \lambda \alpha ́ \delta \alpha, \mathrm{A} \theta \dot{v} v \alpha$ 2001, 168-172.

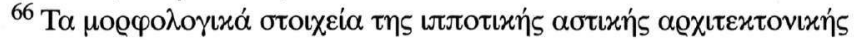

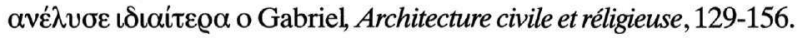




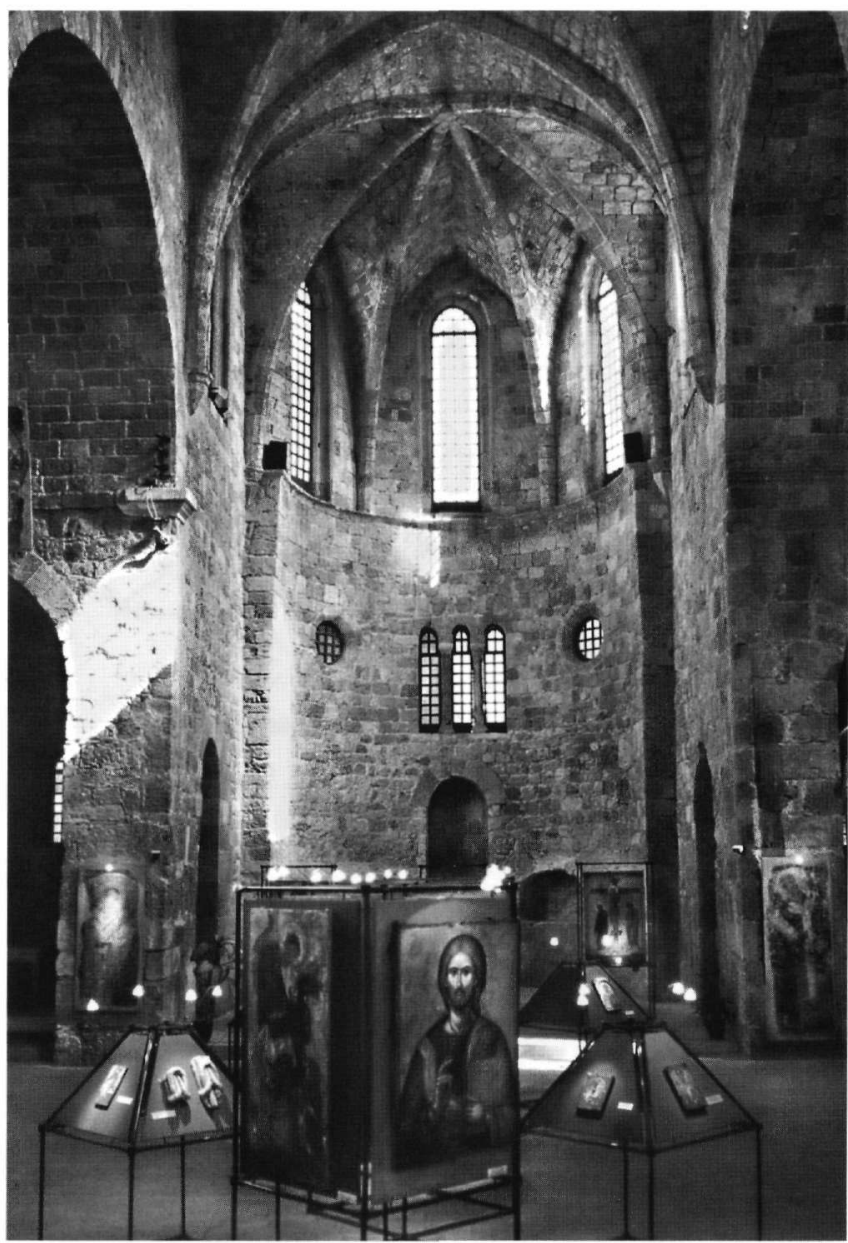

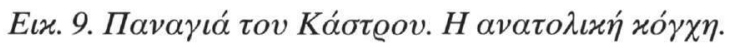

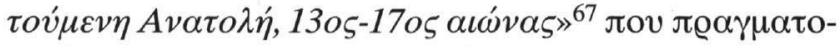

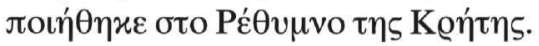

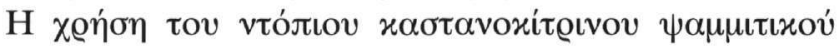

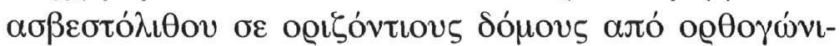

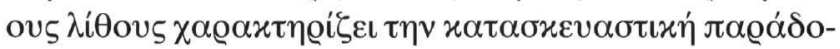

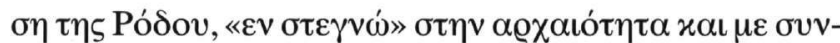

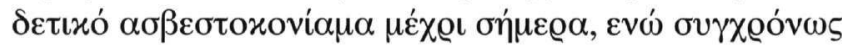

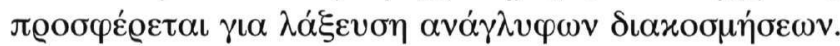

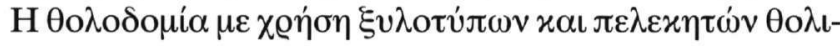

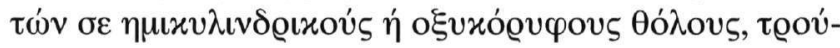

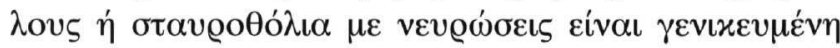

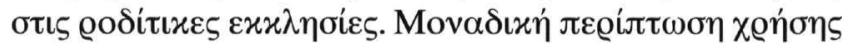

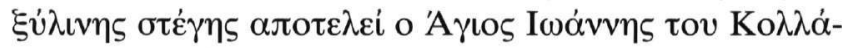

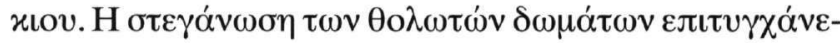

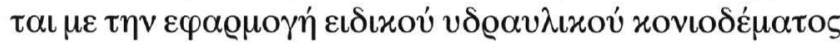

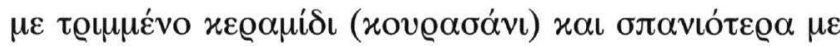

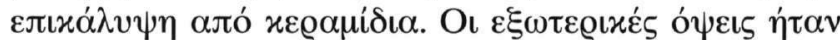

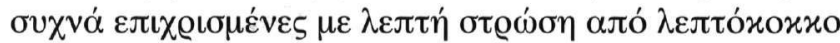

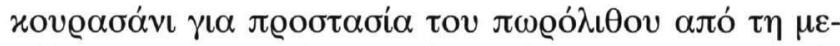

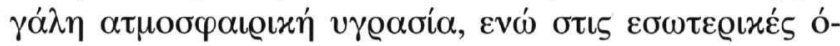

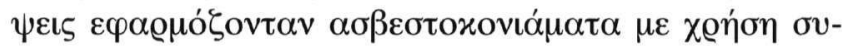

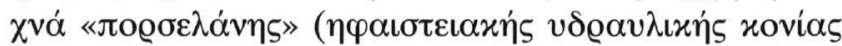

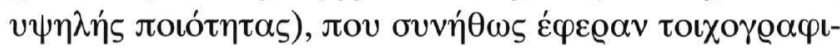

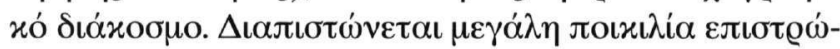

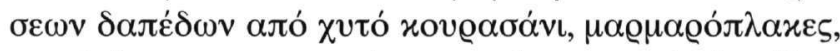

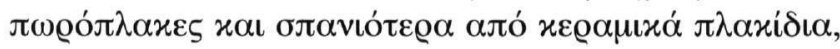

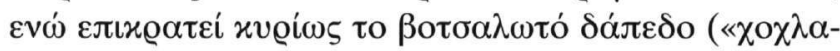

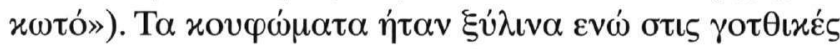

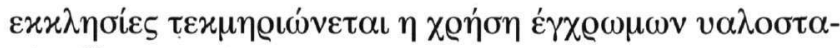

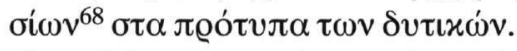

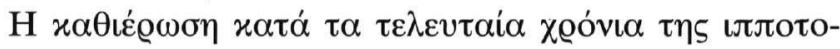

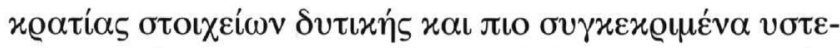

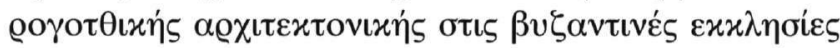

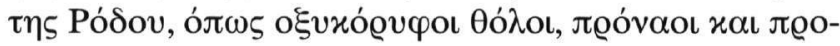

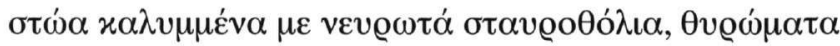

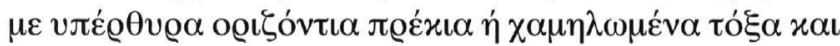

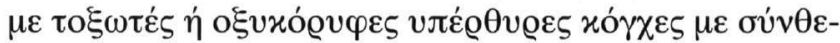

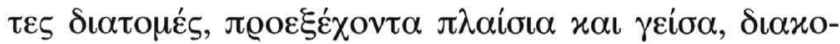

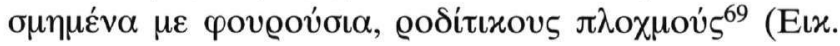

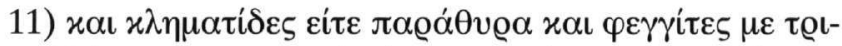

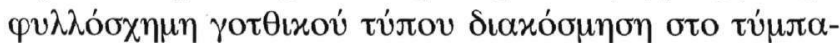

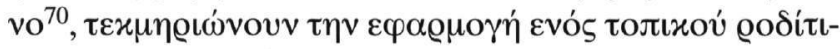

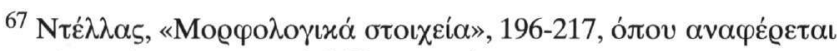

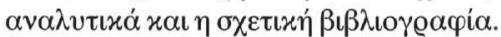

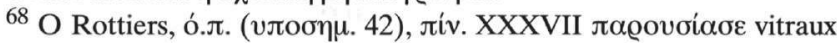

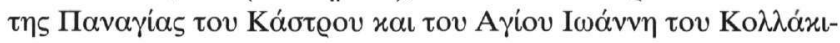

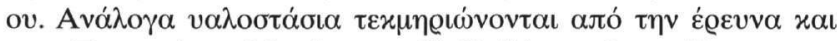

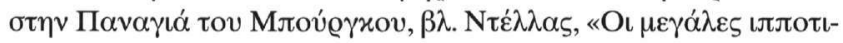

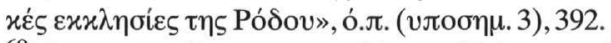

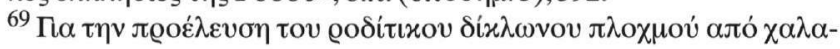

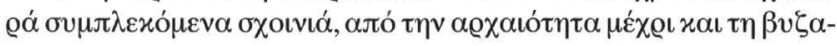

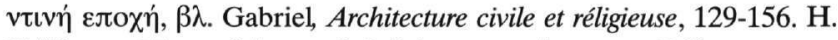

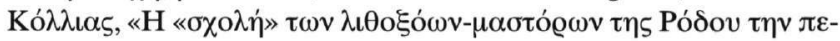

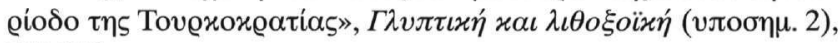
247-249.

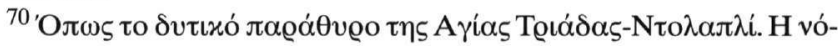

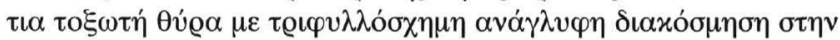

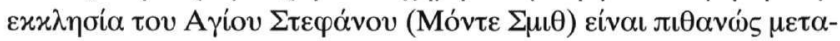

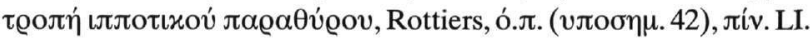




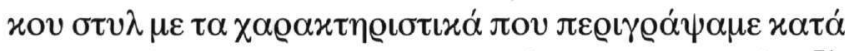

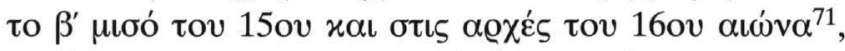

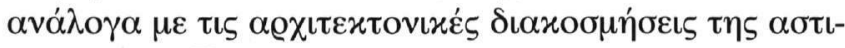

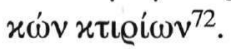

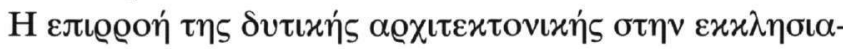

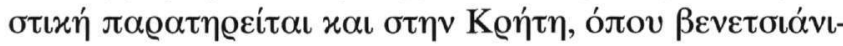

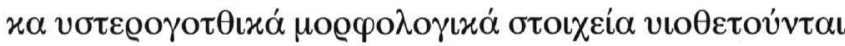

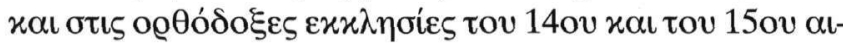

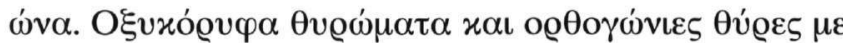

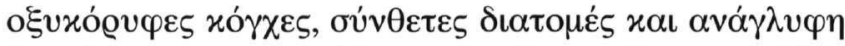

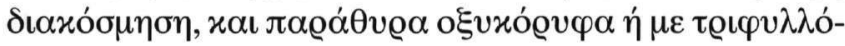

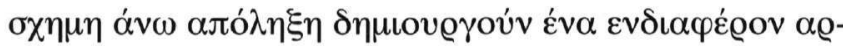

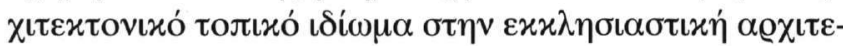

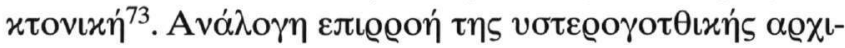

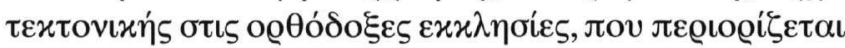

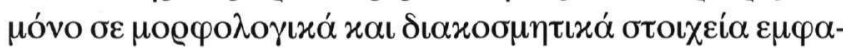

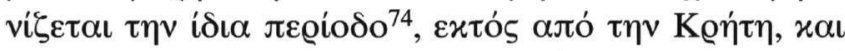

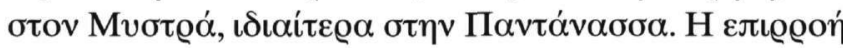

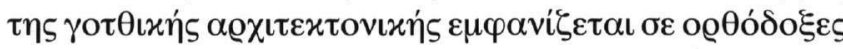

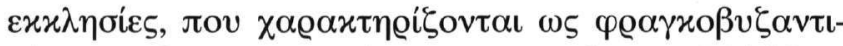

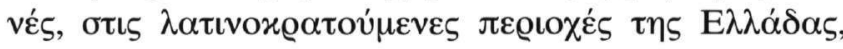

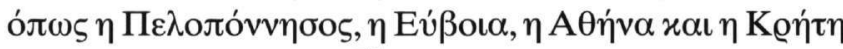

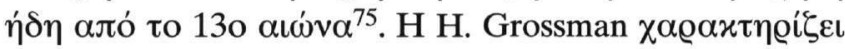

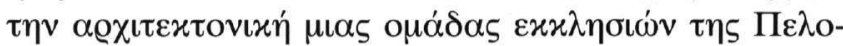

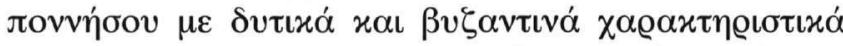

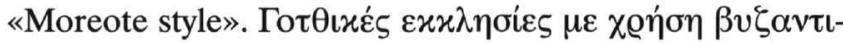

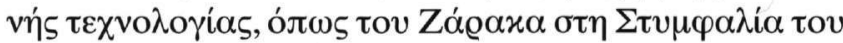

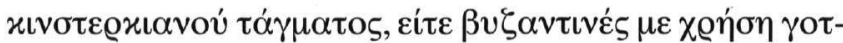

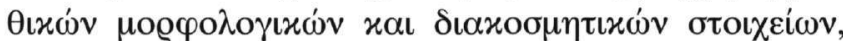

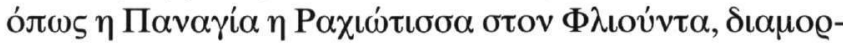

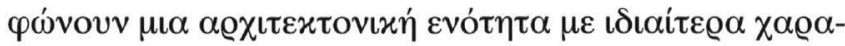

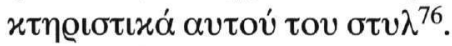

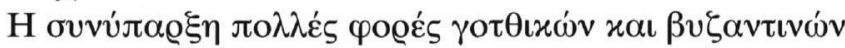

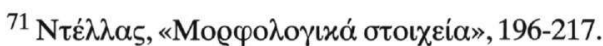

${ }^{72}$ Gabriel, Architecture civile et réligieuse, 1-128.

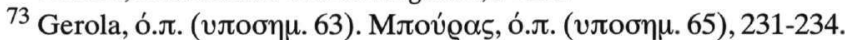

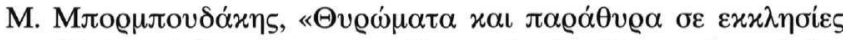

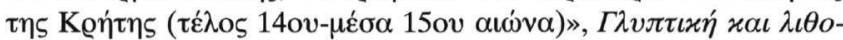

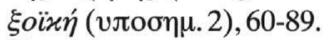

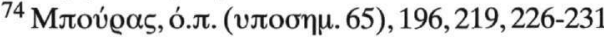

75 'O. $\pi$., 167-175.

${ }^{76}$ H. Grossman, Building Identity: Architecture as Evidence of Cultural Interaction between Latins and Byzantines in Medieval Greece, University

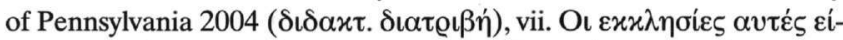

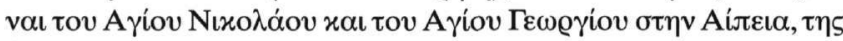

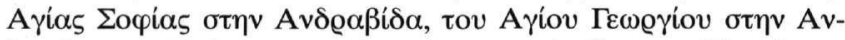

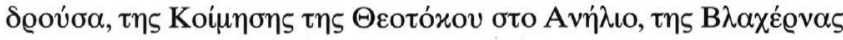

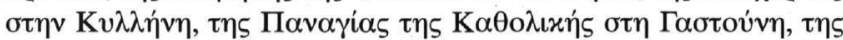

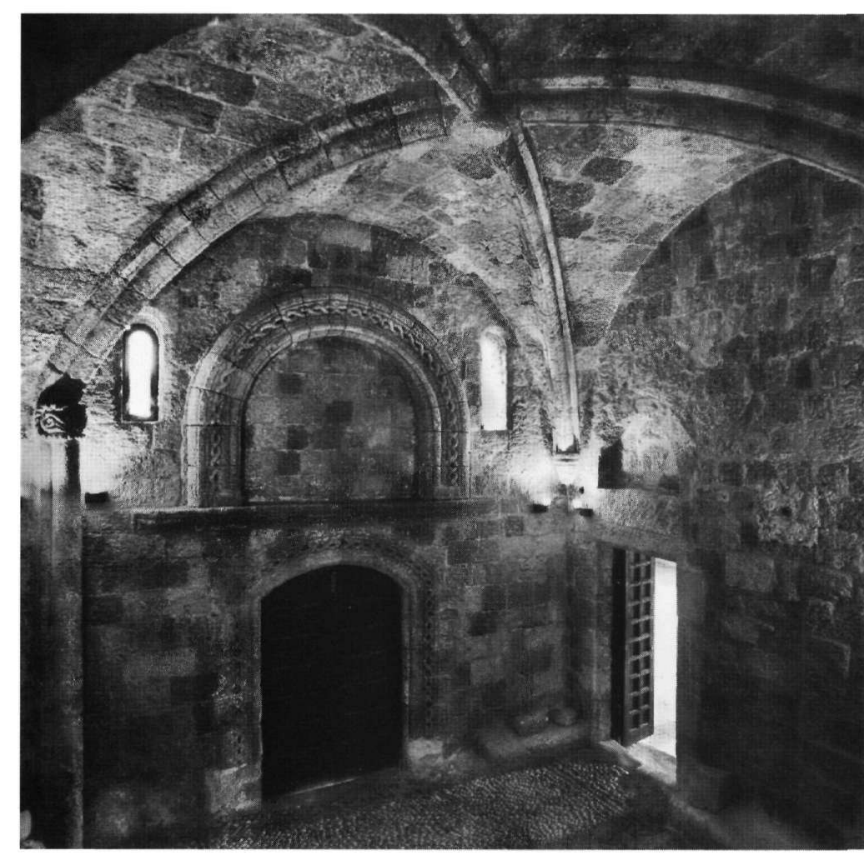

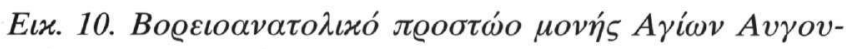

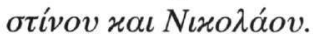

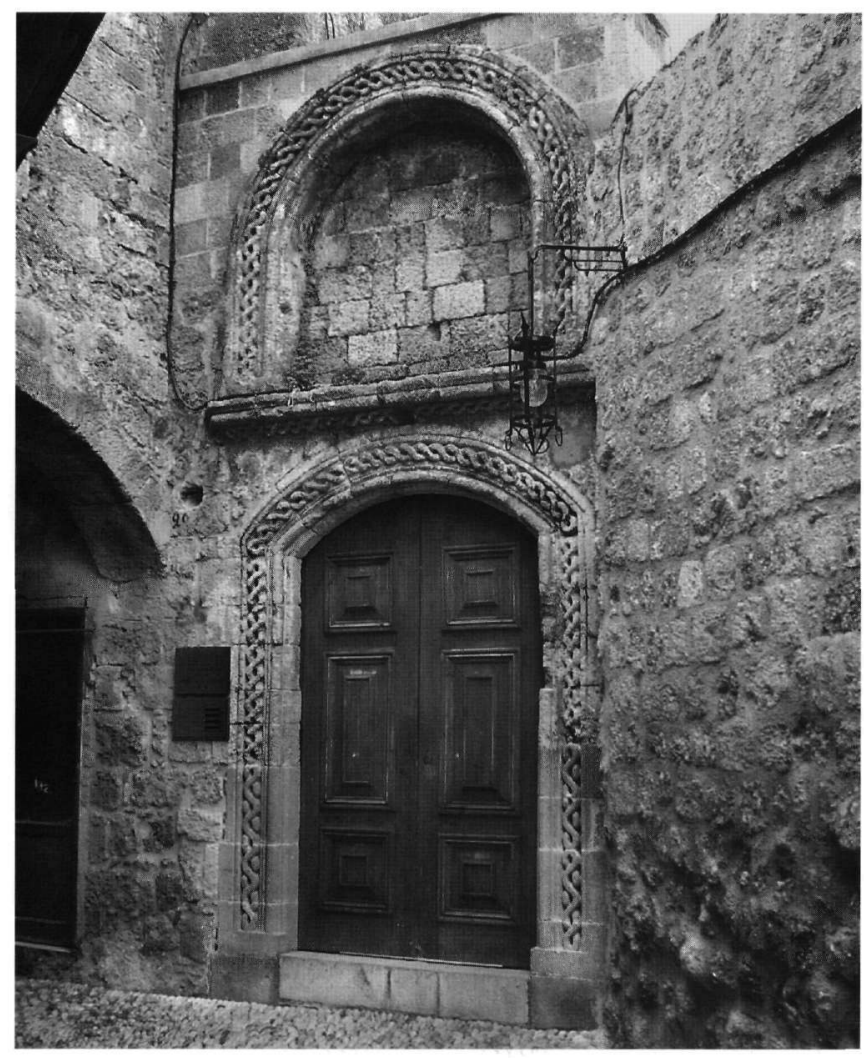

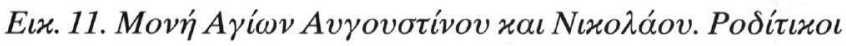

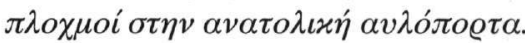




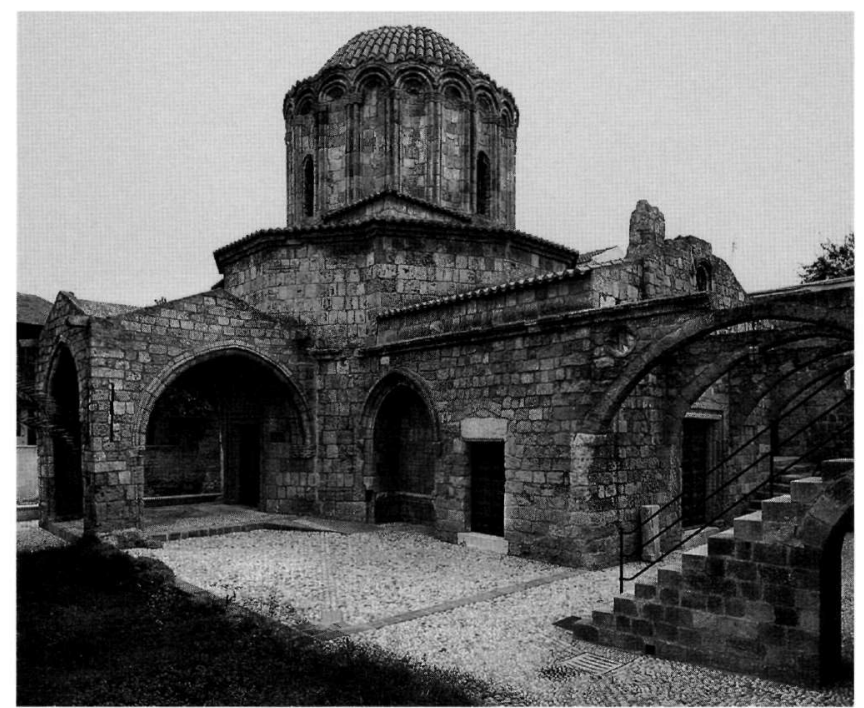

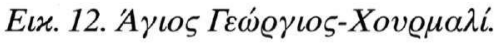

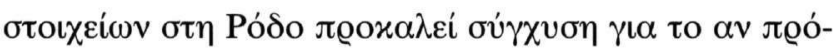

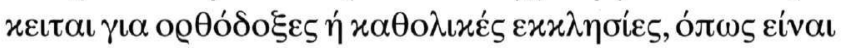

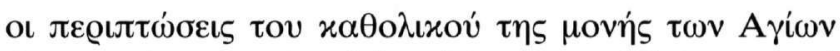

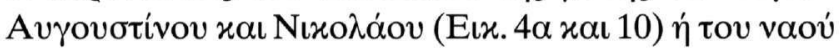

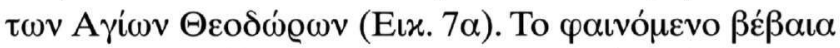

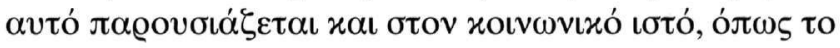

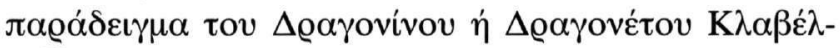

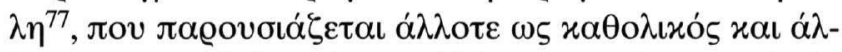

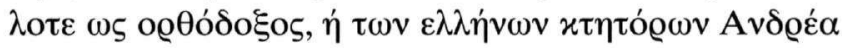

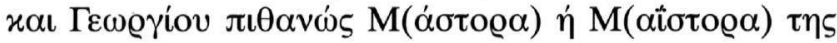

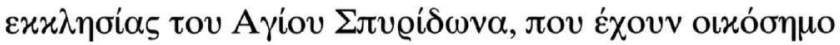

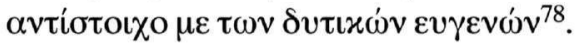

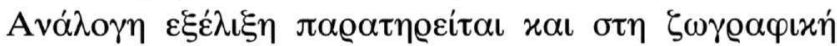

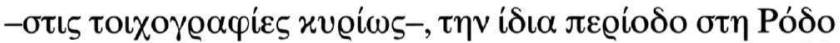

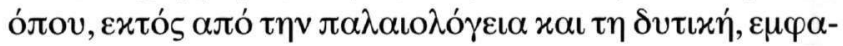

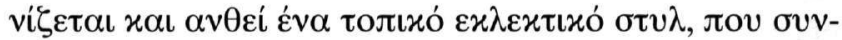

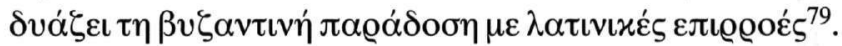

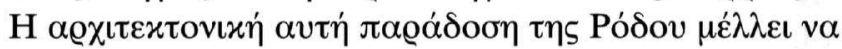

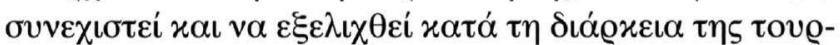

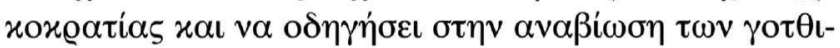

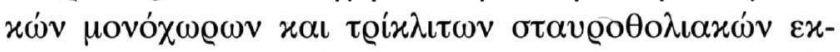

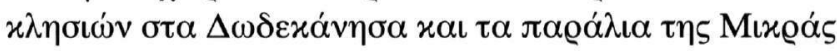

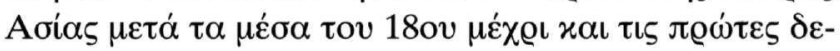

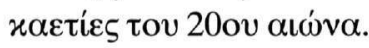

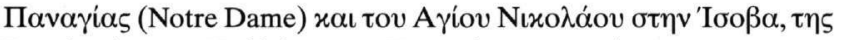

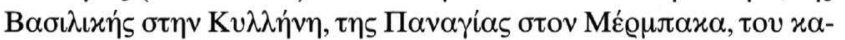

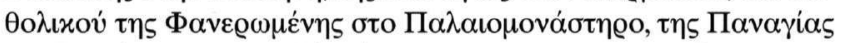

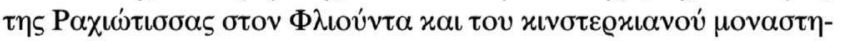

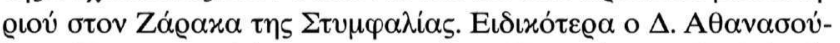

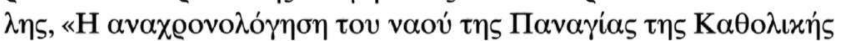

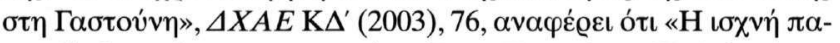

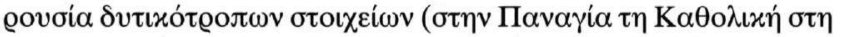

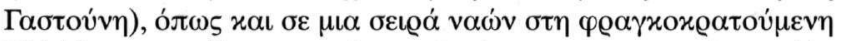

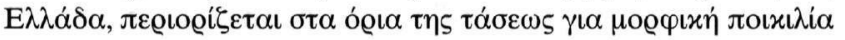

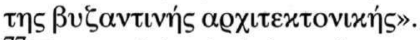

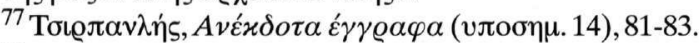

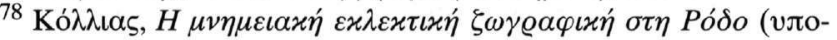

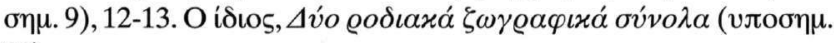
38).

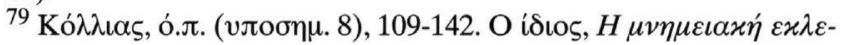

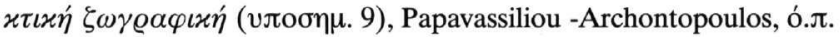

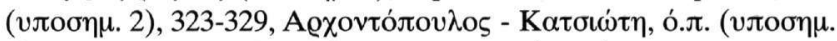

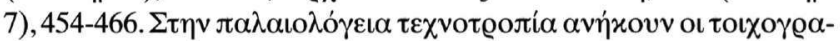

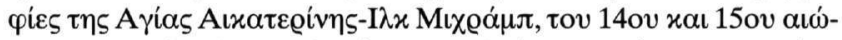

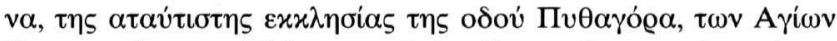

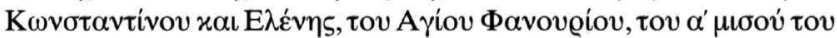
13ov xal 14ov aı

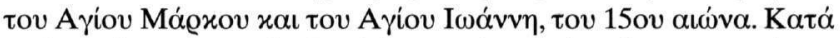

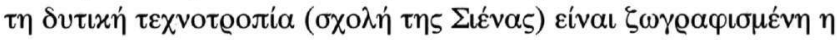

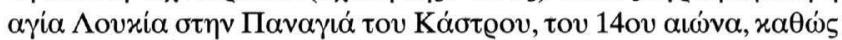

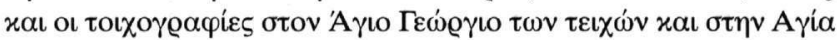

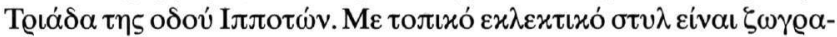

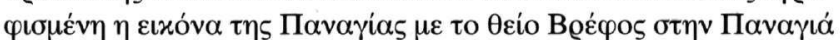

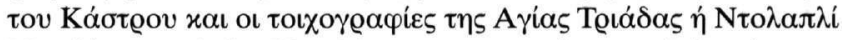

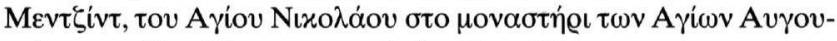

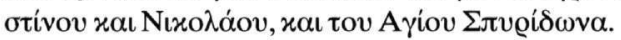




\section{Yorgos Dellas}

\section{THE TYPOLOGY OF RHODIAN CHURCHES DURING THE HOSPITALLER PERIOD (1309-1522)}

\begin{abstract}
$\mathrm{T}$ of the Rhodes town in the Hospitaller period also found expression in the ecclesiastical architecture. The formation of a Late Gothic, later Renaissance, idiom in Rhodian architecture with evident Western influences, in which local materials and traditional Byzantine techniques were used, has yielded some outstanding monuments of this period. The application of the most common Byzantine church types can be widely observed (Fig. 1):

- The three-aisled cross-in-square church with dome, as in the original phase of the Panayia tou Kastrou (Figs $2 \alpha$ and 9) and the Archangel Michael (Demirli Camii) (Fig. 2 2 ).

- The aisleless cross-in-square church with dome, as in the original phase of Ayios Spyridon. This has hitherto been considered a three-aisled church, but careful observation and study of its architectural phases reveals that it was originally an aisleless, cross-in-square domed basilica of the 13th century, which underwent later modifications that gave it the form of a three-aisled cross-in-square domed church at the end of the Hospitaller period (Fig. 3 $\alpha$ ).

- The aisleless barrel-vaulted church, such as Ayios Dimitrios of Piossasco, the church of Ayia Kyriaki, the unidentified church in Kisthiniou Street, the Archangel Michael (Fig. $3 \beta$ ), Ayios Athanasios, Ayia Marina, Ayios Ioannis Prodromos, Ayios Georgios 'ton teichon', the church half incorporated into the fortification wall in the Auvergne section, and the ruined church between Ayia Kyriaki and the Ayioi Theodoroi. It is interesting that chapels were constantly being added to originally aisleless churches, converting them into two-aisled structures, such as the katholikon of the monastery of the Ayioi Augustine and Nikolaos in Omirou Street, which has been restored (Figs $4 \alpha, 10$ and 11), or into three-aisled churches, such as Ayia Aikaterini (Ilk Mihrab) (Fig. 4 $\beta$ ) and Ayioi Konstantinos and Eleni.

- The free-cross church with dome, such as Ayia Paraskevi (Fig. 5 $\alpha$ ), Ayios Artemios (Fig. 5 $\beta$ ), Ayia Triada (Dolapli Camii), the parish church of Ayios Phanourios, the chapel of
\end{abstract}

the Armenopoulos family and the unidentified bombed church in Thiseos Street, next to the hostel of Ayia Aikaterini. To this same type belongs the restored church of Ayios

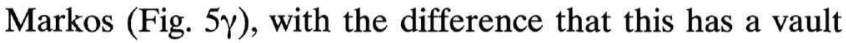
raised above the barrel vault instead of a dome, while the parish church of Ayios Panteleimon was originally barrelvaulted and is now in the type of the free-cross church without a dome, after the addition of a south and north transept with low vaults.

- The use of the tetraconch church with a dome in Ayios Georgios (Chourmali Medrese) in Rhodes town is a special case in Greece (Figs 6 and 12).

The existence of pointed vaults of Gothic origins in the majority of churches with Byzantine typology attests to the direct influence of Western architecture and places their construction during the two centuries of Hospitaller rule in this area. The most important example of this coexistence is the impressive Byzantine cathedral of the Panayia tou Kastrou, in which the roofing of the central aisle has been converted into Gothic cross-vaults (Figs $2 \alpha$ and 9). The addition of open porches, too, usually roofed with ribbed cross-vaults, was adopted by the Orthodox population as early as the Hospitaller period and lends a particular grace to the Byzantine churches in the medieval town.

The most important examples of Western architecture are three-aisled Gothic basilicas, such as Ayios Ioannis tou Kol-

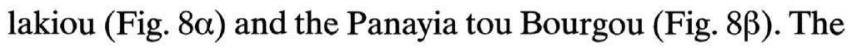
nave of the first of these has a timber roof, while the transept and rectangular sanctuary apse were roofed with Gothic ribbed cross-vaults. The Panayia tou Bourgou was covered with Gothic cross-vaults and the three five-sided apses were roofed with radiating cupolas and ribs. A column and the underground crypt survive of the earlier church, above which the church of the Ayioi Apostoloi was later built. Aisleless churches roofed with a ribbed cross-vault and with a five-sided sanctuary apse covered with radiating ribbed cupolas, include the excavation of the 'Panayia tis Nikis' 
(Fig. 7 3 ) and the church of the Ayioi Apostoloi while the church of the Ayioi Theodoroi (Fig. $7 \alpha$ ) has an apse that is three-sided externally, but covered with a semidome.

Unfortunately none of the large Gothic churches of Rhodes is today preserved intact, and can only be reconstructed through drawings. Despite the fact that the scale and archi- tectural quality of these churches is greatly inferior to Western cathedrals, they exhibit several similarities with corresponding Gothic churches in the eastern Mediterranean, Cyprus, Crete, Ayia Sophia at Andravida in the Peloponnese, and Ayia Paraskevi in Chalkida. 